ARTICLE

\title{
Schizophrenia-associated SAP97 mutations increase glutamatergic synapse strength in the dentate gyrus and impair contextual episodic memory in rats
}

Yuni Kay ${ }^{1}$, Linda Tsan', Elizabeth A. Davis², Chen Tian', Léa Décarie-Spain², Anastasiia Sadybekov ${ }^{3}$, Anna N. Pushkin ${ }^{1}$, Vsevolod Katritch (10 ${ }^{3,4}$, Scott E. Kanoski (iD ${ }^{1,2}$ \& Bruce E. Herring (1D ${ }^{1,5 凶}$

Mutations in the putative glutamatergic synapse scaffolding protein SAP97 are associated with the development of schizophrenia in humans. However, the role of SAP97 in synaptic regulation is unclear. Here we show that SAP97 is expressed in the dendrites of granule neurons in the dentate gyrus but not in the dendrites of other hippocampal neurons. Schizophrenia-related perturbations of SAP97 did not affect CA1 pyramidal neuron synapse function. Conversely, these perturbations produce dramatic augmentation of glutamatergic neurotransmission in granule neurons that can be attributed to a release of perisynaptic GluA1-containing AMPA receptors into the postsynaptic densities of perforant pathway synapses. Furthermore, inhibiting SAP97 function in the dentate gyrus was sufficient to impair contextual episodic memory. Together, our results identify a cell-type-specific synaptic regulatory mechanism in the dentate gyrus that, when disrupted, impairs contextual information processing in rats.

\footnotetext{
${ }^{1}$ Neuroscience Graduate Program, University of Southern California, Los Angeles, CA 90089, USA. ${ }^{2}$ Department of Biological Sciences, Human and Evolutionary Biology Section, Dornsife College of Letters, Arts and Sciences, University of Southern California, Los Angeles, CA 90089, USA. ${ }^{3}$ Department of Chemistry, University of Southern California, Los Angeles, CA 90089, USA. ${ }^{4}$ Quantitative and Computational Biology, University of Southern California, Los Angeles, CA 90089, USA. ${ }^{5}$ Department of Biological Sciences, Neurobiology Section, Dornsife College of Letters, Arts and Sciences, University of Southern California, Los Angeles, CA 90089, USA. 凶email: bherring@usc.edu
} 
S chizophrenia is a debilitating psychiatric disorder that affects around 20 million people worldwide. Symptoms of this disorder include hallucinations, delusions, flat affect, the loss of a sense of personal identity, poor executive function, and deficits in memory ${ }^{1,2}$. Despite many years of research, it remains unclear which cellular and molecular mechanisms in the brain are disrupted in individuals with schizophrenia. It is also unclear which specific regions in the brain are strongly affected by the disruption of these mechanisms. Accumulating evidence supports a connection between SAP97 loss of function and schizophrenia ${ }^{3-14}$. For example: the gene encoding SAP97, DLG1, was recently identified as a potential hub of schizophrenia-related synaptic dysfunction ${ }^{13}$; missense mutations in SAP97 have been identified in individuals with schizophrenia ${ }^{12,14}$; and microdeletion mutations in humans resulting in the loss of a DLG1 allele give rise to a 40 -fold increase in the risk of developing schizophrenia $^{8,9}$. SAP97 is a Membrane-Associated Guanylate Kinase (MAGUK) protein. MAGUK protein family members include PSD-95, PSD-93, SAP102, and SAP97. PSD-95, PSD-93, and SAP102 are major constituents of the glutamatergic synapse postsynaptic density (PSD). At the postsynaptic side of glutamatergic synapses, PSD-95, PSD-93, and SAP102 provide scaffolds for synaptic protein complexes via their three PSD-95/Dlg/ ZO1 (PDZ) domains ${ }^{15}$. It is well established that PSD-95, PSD93, and SAP102 use their PDZ domains to anchor AMPA and NMDA receptors (AMPARs and NMDARs) in the PSD in direct opposition to regions of presynaptic glutamate release. This MAGUK protein-mediated glutamate receptor positioning is critical for efficient activation of these receptors by presynaptically-released glutamate.

In contrast to other MAGUK proteins, the role of SAP97 in synaptic regulation is uncertain, despite the fact that SAP97 is the only MAGUK that can interact directly with AMPARs. This interaction occurs through SAP97's PDZ2 domain binding to the C-terminal PDZ-binding domain of the AMPAR subunit GluA1 ${ }^{16-20}$. It is also unclear whether the C-tail of GluA1 plays any role in synaptic regulation ${ }^{18,21}$. Previous studies have provided conflicting data regarding SAP97's influence on synaptic function $^{22-27}$. Most of these studies have relied on recombinant expression of SAP97 and/or dissociated neuron cultures where synapses form between unknown neuron subtypes. However, one particularly notable study has shown that knocking out SAP97 led to no alteration in glutamatergic neurotransmission in hippocampal CA1 pyramidal neurons ${ }^{28}$. Additionally, mutant mice expressing a PDZ-binding domain lacking the form of the GluA1 subunit which prevents AMPARs from binding to SAP97 (GluA1- $\Delta 7$ mice) were found to have normal glutamatergic neurotransmission in hippocampal CA1 pyramidal neurons ${ }^{18}$. Together, these studies have been used to support a compelling argument against a role for SAP97 in the regulation of glutamatergic synaptic transmission ${ }^{15}$. This lack of understanding regarding SAP97's role in the brain has been particularly frustrating given SAP97's growing implication in schizophrenia, SAP97's similarity to other essential synaptic proteins, and the fact that schizophrenia is largely considered to be a synaptic disease ${ }^{13,14,29}$.

Increasing evidence points to dysfunction of the dentate gyrus as a contributing factor in the development of schizophrenia1,30-38. The dentate gyrus serves as the gateway for information coming into the hippocampus via the perforant pathway, and symptoms associated with schizophrenia exhibit a high degree of correlation with symptoms stemming from dentate gyral dysfunction ${ }^{1,30,39}$. In the present study, we find that the expression of $\beta$ SAP97, the major isoform of SAP97 ${ }^{24}$, is absent in the apical and basal dendrites of CA3 and CA1 pyramidal neurons of the hippocampus. In contrast, robust $\beta$ SAP97 expression is observed in the dendrites of granule neurons in the dentate gyrus (DG granule neurons). Given that reduced SAP97 expression in the brain is associated with a substantially increased risk of developing schizophrenia ${ }^{3,8,9}$, we were interested in whether $\beta$ SAP97 regulates glutamatergic synapse function in DG granule neurons and whether reduced $\beta$ SAP97 expression in the dentate gyrus is sufficient to produce schizophrenia-related behavioral phenotypes in rodents.

Here we find that reducing $\beta$ SAP97 expression in DG granule neurons results in a dramatic increase in AMPAR-mediated synaptic transmission following perforant path stimulation. Conversely, inhibition of $\beta$ SAP97 expression in CA1 pyramidal neurons had no effect on glutamatergic synaptic transmission. Furthermore, we find that reducing 3 SAP97 expression specifically within the dentate gyrus is sufficient to disrupt contextual episodic memory processing in rats. Similar memory deficits are present in individuals with schizophrenia and have been proposed to contribute to the development of delusions, disorganization, hallucinations, and the loss of a sense of personal identity observed with this disorder ${ }^{40-52}$. Finally, we show that schizophrenia-related missense mutations clustered in SAP97's PDZ2 domain also produce large increases in synaptic AMPAR function in DG granule neurons that can be attributed to the release of perisynaptic GluA1-containing AMPARs into the PSDs of perforant pathway synapses. Altogether, our study identifies a cell-type-specific synaptic regulatory mechanism in the dentate gyrus that, when disrupted, impairs contextual episodic memory in rats.

\section{Results}

BSAP97 knockdown augments synaptic AMPAR-mediated neurotransmission in DG granule neurons. Both dentate gyral and SAP97 dysfunction have been implicated in the development of schizophrenia. To determine where BSAP97 is endogenously expressed in the rat hippocampus, we performed an immunohistochemical analysis of $\beta$ SAP97 expression in hippocampal slices from rats. Remarkably, this analysis revealed robust expression of BSAP97 in the molecular layer of the dentate gyrus but not in the stratum radiatum or stratum oriens of CA3 and CA1 (Fig. 1a). BSAP97 expression overlapped with the dendriticmarker MAP2 in the molecular layer of the dentate gyrus, indicating that SSAP97 is expressed in the dendrites of DG granule neurons (Fig. 1a and Supplementary Fig. 1a). We validated this experiment using a second SAP97 antibody, which again displayed dendritic labeling that was specific to DG granule neurons (Supplementary Fig. 1b). An isotype control primary antibody, as well as the secondary antibody alone, produced no visible signal in our slices (Supplementary Fig. 1c). An immunizing peptide blocking experiment was also performed to further validate the specificity of our immunolabeling, and again no visible signal was found with the peptide blocked sample, demonstrating that the immunolabeling was specific (Supplementary Fig. 1c). Together, these results suggest that glutamatergic perforant pathway synapses between presynaptic entorhinal cortical neurons and postsynaptic DG granule neurons may be selectively regulated by BSAP97 in DG granule neurons. To determine whether $\beta$ SAP97 plays a role in DG granule neuron glutamatergic synapse regulation, we generated an RNAi against $\beta$ SAP97. AAV-mediated expression of our $\beta$ SAP97-microRNA (miR) construct in dissociated rat hippocampal neurons reduced endogenous $\beta S A P 97$ protein levels by $\sim 75 \%$ with no effects observed on the expression of other MAGUK proteins (Fig. 1b). Using our miR construct, we knocked down the expression of $\beta$ SAP97 in DG granule neurons in rat organotypic entorhino-hippocampal slice cultures through biolistic transfection ${ }^{23,53}$. Biolistic transfection of these slices allows for sparse transfection of neurons maintained within their 
a

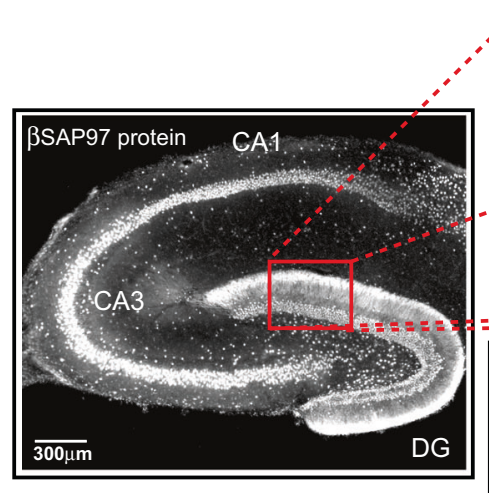

C

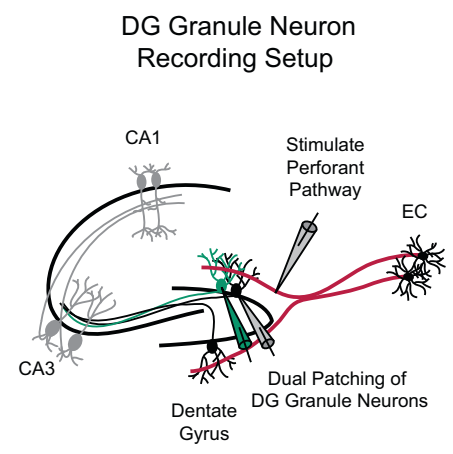

f

BSAP97-miR

Surface AMPAR Current DG Granule Neurons
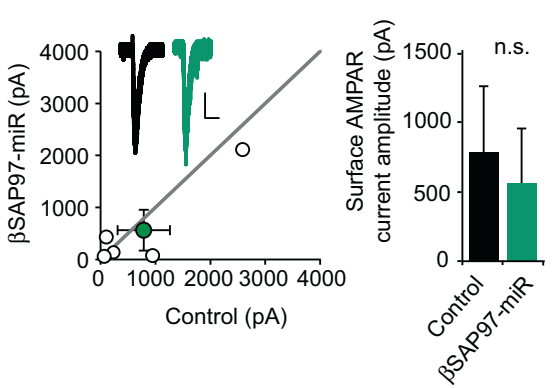

b

Specificity of $\beta S A P 97$ Knockdown in Hippocampal Neurons

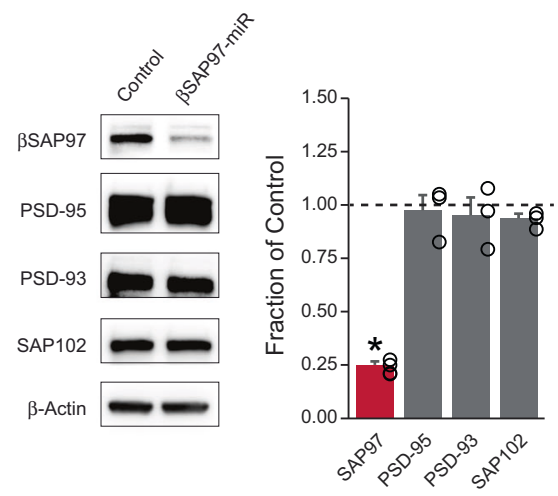

e
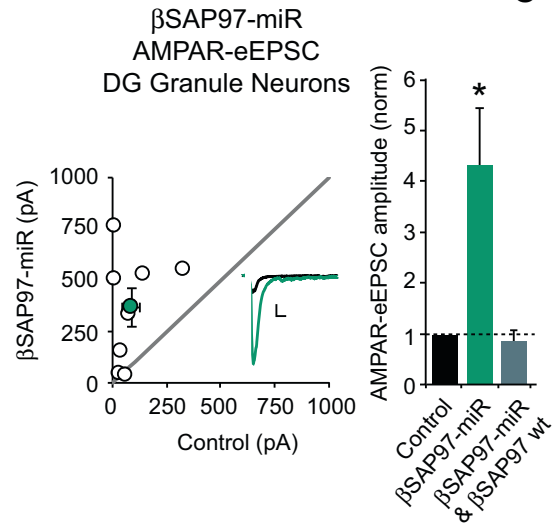

g BSAP97-miR AMPAR-eEPSC Rectification DG Granule Neurons

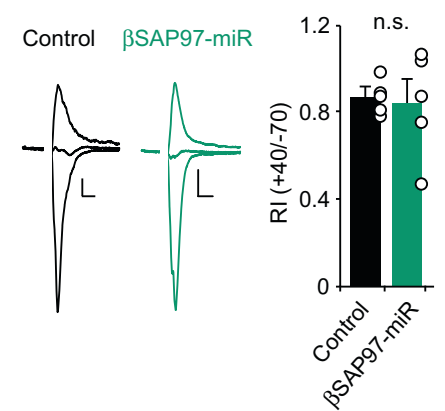

BSAP97-miR DG Granule Neurons
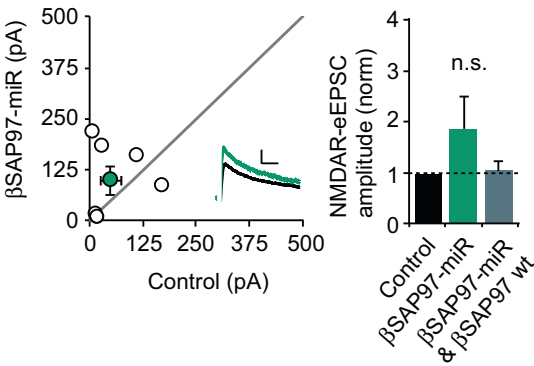

h

BSAP97-miR GABAR-eIPSC DG Granule Neurons
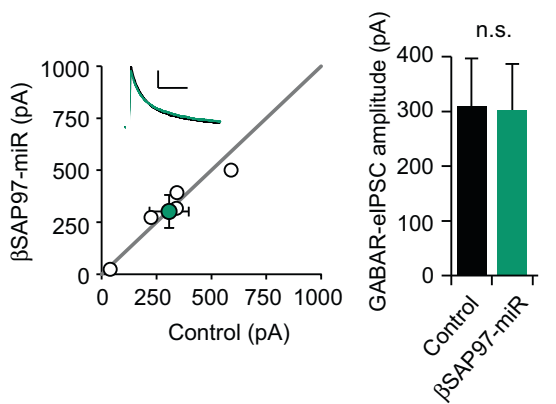
NMDAR-eEPSC intact, endogenous circuitry ${ }^{54-59}$. Six days after transfection, recordings of AMPA receptor- and NMDA receptor-evoked excitatory postsynaptic currents (AMPAR- and NMDAReEPSCs) were made from fluorescent 3 SAP97-miR transfected DG granule neurons and neighboring control neurons simultaneously during perforant pathway stimulation (Fig. 1c). This approach allows for a pair-wise, internally controlled comparison of the consequences of acute genetic manipulations that are limited to the postsynaptic neuron ${ }^{54-59}$. Knocking down $\beta$ SAP97 in DG granule neurons using this approach led to a striking 4-fold increase in AMPAR-eEPSC amplitudes compared to paired control neurons (Fig. 1d), a surprising phenotype given that knocking down traditional MAGUK proteins reduces AMPAR-eEPSC amplitudes in CA1 pyramidal neurons ${ }^{60}$. A significant change in NMDAR-eEPSC amplitude was not observed following $\beta$ SAP97 knockdown in DG granule neurons (Fig. 1e). RT-PCR analysis confirmed that the increase in AMPAR-eEPSC amplitude we observed was not due to a secondary upregulation of aSAP97 expression (Supplementary Fig. 2a). aSAP97 protein is not endogenously expressed in the hippocampus ${ }^{24}$ but has been shown to augment synaptic function when exogenously expressed in neurons ${ }^{61}$. To further verify that the 4-fold increase in AMPAR-eESPC amplitude was indeed due to knockdown of $\beta$ SAP97 specifically, we generated a recombinant, RNAi-resistant $\beta$ SAP97 expression construct (Supplementary Fig. 2b). We first verified that the expression of this RNAiresistant $\beta$ SAP97 was not inhibited by our $\beta$ SAP97-miR (Supplementary Fig. 2c). Next, we co-expressed our $\beta$ SAP97-miR with an mCherry-tagged RNAi-resistant $\beta S A P 97$, and verified via imaging that this recombinant $\beta$ SAP97 localized to dendritic 
Fig. 1 ßSAP97 knockdown augments AMPAR-mediated neurotransmission in DG granule neurons. a (Left) Representative immunolabeling of $\beta$ SAP97 in a rat entorhino-hippocampal slice. Red box shows enlarged portion of the dentate gyrus. GL, granule layer; ML, molecular layer. (Right) Co-immunolabeling of MAP2 and $\beta$ SAP97 in dendrites of DG granule neurons. b Western blot analysis showing specificity of $\beta S A P 97-m i R$ in dissociated hippocampal neurons. $\beta S A P 97-m i R$ reduces $\beta$ SAP97 protein expression in dissociated hippocampal neurons without altering PSD-95, PSD-93, and SAP102 protein expression. (Right) Bar graph shows quantification of SAP97, PSD-95, PSD-93, and SAP102 protein expression following $\beta$ SAP97-miR-mediated knockdown. $\mathrm{n}$ represents independent experiments. ( $\beta$ SAP97, $n=4, p=0.00002$; PSD-95, $n=3, p=0.75$; PSD-93, $n=3, p=0.62 ;$ SAP102, $n=3, p=0.09 ;$ two sample $T$ tests). c Schematic representation of electrophysiological recording setup for DG granule neurons. For panels (d) and (e), open circles are single pairs of control and transfected neurons, filled circles represent the mean amplitudes $( \pm S E M)$, insets show representative current traces from control (black) and $\beta$ SAP97-miR transfected (green) neurons with stimulation artifacts removed. Scale bars: 20 ms for AMPA, 50 ms for NMDA, 100pA. Bar graphs show the average AMPAR-eEPSC and NMDAR-eEPSC amplitudes ( \pm SEM) of DG granule neurons expressing the $\beta$ SAP97-miR (green) and DG granule neurons co-expressing the $\beta$ SAP97-miR and miR-resistant wild-type (wt) $\beta S A P 97$ cDNA (gray) normalized to their respective control cell average eEPSC amplitudes (black). $\beta$ SAP97-miR expression increases AMPAR-eEPSC amplitude in DG granule neurons $(n=8$ pairs, $p=0.019$, paired $T$ test) (d) but has no detectable effect on NMDAR-eEPSC amplitude ( $n=7$ pairs, $p=0.27$, paired $T$ test) (e). Co-expression of $\beta$ SAP97-miR and miR-resistant $\beta S A P 97$ cDNA has no detectable effects on either AMPAR-eEPSC ( $n=8$ pairs, $p=0.227$, paired $T$ test) (d) or NMDAR-eEPSC amplitudes $(n=8$ pairs, $p=0.99$, paired $T$ test) (e). Additional details of the rescue experiment are provided in Supplementary Fig. 2b, c, e. f $\beta S A P 97-m i R$ expression does not change surface AMPAR current amplitude in DG granule neurons. (Left) Open circles in the scatter plot represent single pairs of control and transfected neurons, filled circles represent the mean amplitudes $( \pm$ SEM), insets show representative surface AMPAR current traces from control (black) and transfected (green) neurons. Scale bars: $5 \mathrm{~s}, 25 \mathrm{pA}$. (Right) Bar graph shows average surface AMPAR current amplitudes ( \pm SEM) of control (black) and $\beta S A P 97-m i R$ expressing (green) DG granule neurons ( $n=5$ pairs, $p=0.335$, paired $T$ test). $\mathbf{g}$ BSAP97 knockdown does not change AMPAR-eEPSC rectification. (Left) Representative current traces, scale bars: 20 ms, 20 pA. (Right) Bar graph shows mean \pm SEM of the AMPAR-eEPSC rectification index of control (black, $n=5$ neurons) and $\beta$ SAP97-miR expressing (green, $n=5$ neurons) DG granule neurons ( $p=0.81$, two sample $T$ test). $\mathbf{h} \beta$ SAP97 knockdown does not affect GABAergic synapse function in DG granule neurons. Open circles are single pairs of control and transfected neurons, filled circles represent the mean amplitudes $( \pm$ SEM), insets show representative current traces from control (black) and $\beta S A P 97-m i R$ transfected (green) neurons with stimulation artifacts removed. Scale bars: 100pA, $100 \mathrm{~ms}$. Bar graph shows the average GABAR-eIPSC amplitudes ( \pm SEM) of DG granule neurons expressing the $\beta$ SAP97-miR (green) and control DG neurons (black) ( $n=5$ pairs, $p=0.81$, paired $T$ test). ${ }^{*} p<0.05$; n.s., not significant. All statistical tests performed were two-tailed. Source data are provided in the Source Data file.

spines, consistent with previous work demonstrating perisynaptic localization of $\beta S A P 97^{61}$ (Supplementary Fig. 2d). Finally, we coexpressed our $\beta S A P 97-\mathrm{miR}$ and the RNAi-resistant $\beta$ SAP97 construct in DG granule neurons and found that expression of the RNAi-resistant $\beta$ SAP97 returned AMPAR-eEPSC amplitude to wild-type levels (Fig. 1d and Supplementary Fig. 2e), confirming the specificity of our genetic manipulation.

The increase in synaptic AMPAR-eEPSC amplitude we observe following knockdown of BSAP97 may arise from increased exocytosis of intracellular AMPARs or rearrangements of AMPARs already on surface of neurons that result in more AMPARs in the PSD of glutamatergic synapses. To determine whether 3 SAP97 supports intracellular AMPAR stores or inhibits surface AMPARs from entering the PSD, we puffed glutamate onto the dendrites of neighboring whole-cell patch clamped control and 3 SAP97-miR-expressing DG granule neurons. Puffing glutamate onto the dendrites of these neurons produced currents stemming from the activation of extrasynaptic, perisynaptic, as well as synaptic AMPARs on the surface of dendrites. This approach allowed us to compare the number of AMPARs on the dendritic surface of neurons in the presence and absence of BSAP97. We found that knocking down $\beta$ SAP97 produced no change in AMPAR surface current amplitude in DG granule neurons (Fig. 1f). These data are consistent with that of previous work demonstrating that recombinant $\beta$ SAP97 restricts the ability of perisynaptic AMPARs on the spine surface from accessing the PSD of glutamatergic synapses ${ }^{61}$.

Given that $\beta$ SAP97 likely prevents a subpopulation of AMPARs from reaching synapses, we were interested in whether $\beta S A P 97$ associates with AMPARs that have a subunit composition distinct from synaptic AMPARs. Previous studies have reported the presence of calcium-permeable GluA2 subunitlacking AMPARs in neurons that are made available to synapses during activity-dependent synaptic potentiation ${ }^{62}$. One possibility is that $\beta$ SAP97 binds to and holds GluA2-lacking AMPARs away from glutamatergic synapses in DG granule neurons, and that knocking down $\beta$ SAP97 releases these receptors, allowing them to reach synapses. To test this hypothesis, we compared synaptic AMPAR rectification in control neurons to neurons expressing our 3 SAP97-miR. GluA2-lacking AMPARs exhibit inwardly rectifying currents whereas GluA2-containing AMPARs have a linear current/voltage relationship. We found that knocking down BSAP97 had no effect on +40/-70 AMPAR-eEPSC amplitude ratios, with both control neurons and $\beta$ SAP97 knockdown neurons exhibiting linear $+40 /-70 \mathrm{mV}$ AMPAR-eEPSC amplitude ratios characteristic of GluA2-containing AMPARs (Fig. 1g). Thus, we conclude that knocking down $\beta$ SAP97 does not result in the insertion of GluA2-lacking AMPARs into glutamatergic synapses.

Finally, we performed additional basic characterizations of the effects of $\beta$ SAP97 knockdown on other neuronal and synaptic properties. We determined that knockdown of $\beta$ SAP97 has no effect on the resting membrane potential or the excitability of DG granule neurons (Supplementary Fig. 2f). We also found that ßSAP97 knockdown in DG granule neurons has no effect on GABAergic synapse function (Fig. 1h).

BSAP97 knockdown has no effect on glutamatergic neurotransmission in CA1 pyramidal neurons. Our immunohistochemical analysis revealed no expression of $\beta S A P 97$ in the dendrites of CA1 pyramidal neurons (Fig. 2a and Supplementary Fig. 1b). However, BSAP97 expression was observed in the cell bodies of these neurons. To determine whether inhibiting $\beta$ SAP97 expression affects glutamatergic synaptic transmission in CA1 pyramidal neurons, we transfected these neurons with our BSAP97-miR. We then examined AMPAR- and NMDAR-eEPSC amplitudes in pairs of $\beta$ SAP97-miR transfected and control CA1 pyramidal neurons following Schaffer collateral stimulation (Fig. 2b). As suspected by the lack of $\beta$ SAP97 expression in the dendrites of these neurons, knocking down 3 SAP97 led to no significant effects on either AMPAR- or NMDAR-eEPSC amplitudes (Fig. 2c, d). We also assessed the effects of BSAP97 knockdown on dendritic AMPAR surface current in CA1 

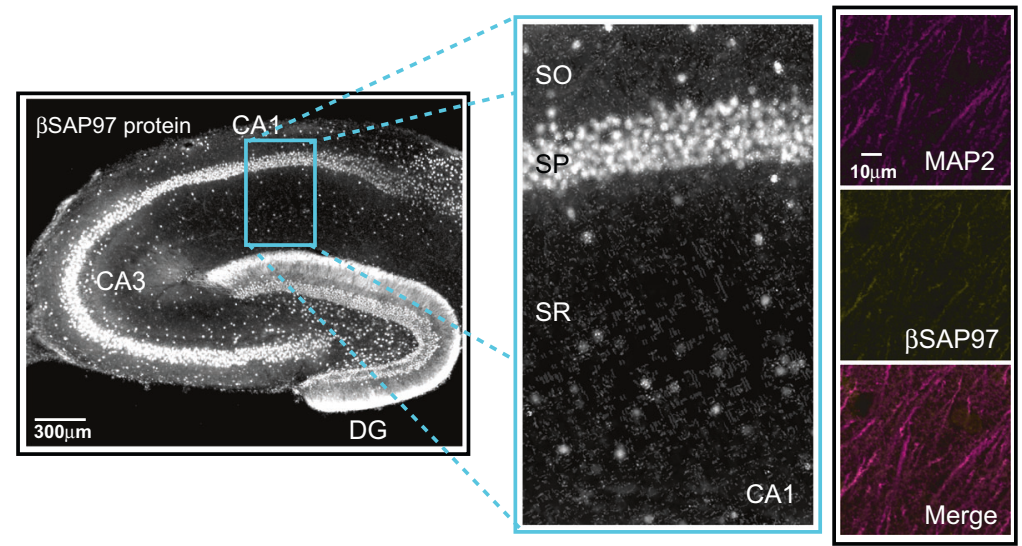

C

BSAP97-miR

AMPAR-eEPSC

CA1 Pyramidal Neurons
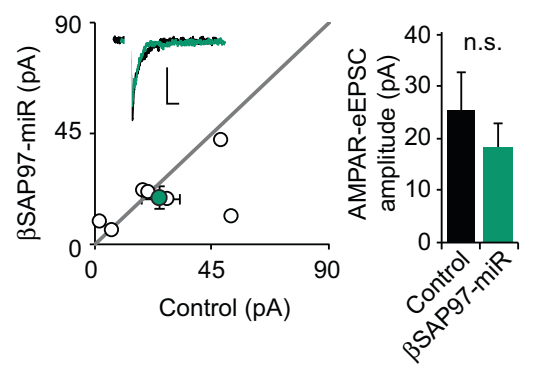

d

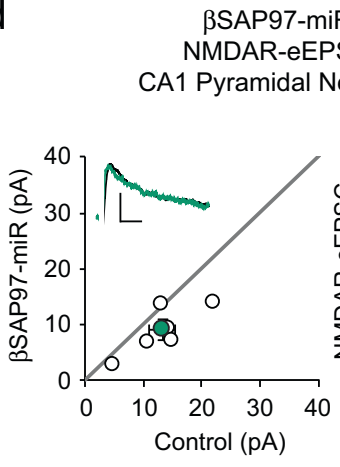

b

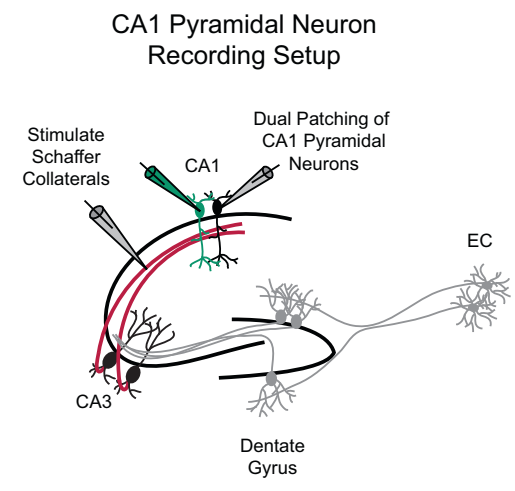

e

BSAP97-miR

Surface AMPAR Current

CA1 Pyramidal Neurons

Fig. 2 及SAP97 knockdown has no effect on glutamatergic neurotransmission in CA1 pyramidal neurons. a (Left) Representative immunolabeling of BSAP97 in a rat entorhino-hippocampal slice. Blue box shows enlarged CA1 region. SO, stratum oriens; SP, stratum pyramidale; SR, stratum radiatum. (Right) Co-immunolabeling of MAP2 and 3 SAP97 in dendrites of CA1 neurons. b Schematic representation of electrophysiological recording setup for CA1 pyramidal neurons. For panels (c) and (d), open circles are single pairs of control and transfected neurons, filled circles represent the mean amplitudes $( \pm$ SEM), insets show representative current traces from control (black) and transfected (green) neurons with stimulation artifacts removed. Scale bars: $20 \mathrm{~ms}$ for AMPA, $50 \mathrm{~ms}$ for NMDA, 20 pA. Bar graphs show the average AMPAR-eEPSC and NMDAR-eEPSC amplitudes ( \pm SEM) of CA1 pyramidal neurons expressing the $\beta S A P 97-m i R$ (green) and control cell average eEPSC amplitudes (black). $\beta S A P 97-m i R$ expression has no detectable effects on either AMPAR-eEPSC ( $n=7$ pairs, $p=0.29$, paired $T$ test, n.s., not significant) (c) or NMDAR-eEPSC amplitudes $(n=6$ pairs, $p=0.29$, paired $T$ test, n.s., not significant) (d) in CA1 pyramidal neurons. e $\beta S A P 97-m i R$ does not change surface AMPAR current amplitude in CA1 pyramidal neurons. (Left) Open circles in the scatter plot represent single pairs of control and transfected neurons, filled circles represent the mean amplitudes ( \pm SEM), insets show representative surface AMPAR current traces from control (black) and transfected (green) neurons. Scale bars: 5 s, 200pA. (Right) Bar graph shows average surface AMPAR current amplitudes ( \pm SEM) of control (black) and $\beta$ SAP97-miR expressing (green) CA1 pyramidal neurons $(n=5$ pairs, $p=0.92$, paired $T$ test). n.s., not significant. All statistical tests performed were two-tailed. Source data are provided in the Source Data file.

pyramidal neurons by puffing glutamate onto the apical dendrites of neighboring whole-cell patch clamped control and BSAP97miR-expressing CA1 pyramidal neurons. We found that $\beta$ SAP97 knockdown does not affect dendritic AMPAR surface current in CA1 pyramidal neurons (Fig. 2e). In further characterizing the effects of $\beta$ SAP97 knockdown in CA1 pyramidal neurons, we stimulated axons within the stratum oriens to evaluate the function of glutamatergic synapses on basal dendrites (Supplementary Fig. 3a) and again found no effect on either AMPAR- or NMDAR-eEPSC amplitudes (Supplementary Fig. 3b, c). Taken together with our results in DG neurons, we have now identified a specific set of glutamatergic synapses where $\beta$ SAP97 plays an essential, cell-type-specific role in regulating glutamatergic neurotransmission.

PSD-95, PSD-93, SAP102 knockdown similarly affects CA1 pyramidal neurons and DG granule neurons. In the hippocampus, our results demonstrate that $\beta$ SAP97 inhibits synaptic
AMPAR function specifically in DG granule neurons. This is in contrast to PSD-95, PSD-93, and SAP102, which are expressed in the dendrites of CA1 and CA3 pyramidal neurons and facilitate synaptic AMPAR and NMDAR function in CA1 pyramidal neurons and many other neurons in the brain ${ }^{15,60,63-68}$. In addition to being expressed in the dendrites of CA1 and CA3 pyramidal neurons, PSD-95, PSD-93, and SAP102 immunolabeling are also present in the molecular layer of the dentate gyrus $^{66-68}$. Therefore, we considered the possibility that all major MAGUK proteins play fundamentally different roles in DG granule neurons compared to what has been conventionally established in CA1 pyramidal neurons. If this was the case, it would suggest that the phenotype we observed in DG granule neurons following $\beta$ SAP97 knockdown is due to general MAGUK function being different in DG granule neurons, rather than BSAP97 itself playing a unique regulatory role at perforant pathDG granule neuron synapses. To test this, we compared the result of knocking down PSD-95, PSD-93, and SAP102 in either CA1 or DG granule neurons by expressing a previously validated chained, 
a

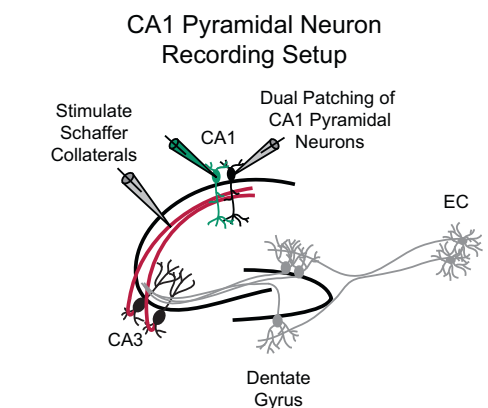

b

PSD-95, -93, SAP102-miRs AMPAR-eEPSC

CA1 Pyramidal Neurons

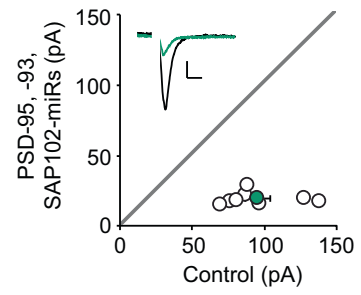

C

PSD-95, -93, SAP102-miRs NMDAR-eEPSC

CA1 Pyramidal Neurons

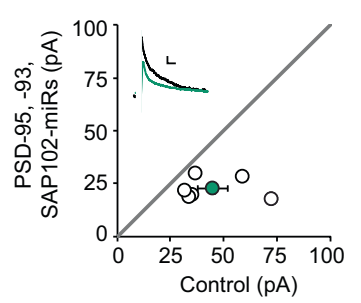

d

e

f

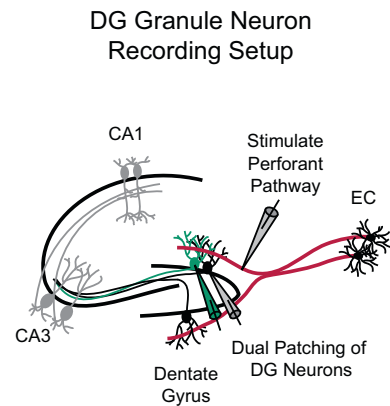

PSD-95, -93, SAP102-miRs AMPAR-eEPSC

DG Granule Neurons
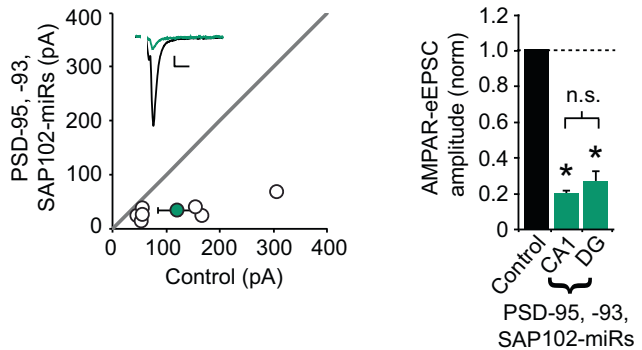

PSD-95, -93, SAP102-miRs NMDAR-eEPSC

DG Granule Neurons

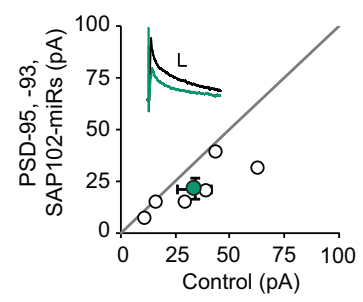

h

NMDAR-eEPSC

Summary

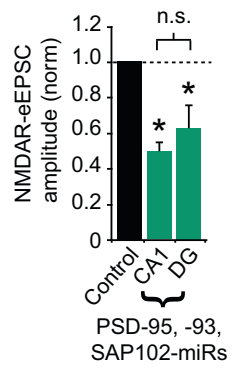

Fig. 3 PSD-95, PSD-93, SAP102 knockdown similarly affects CA1 pyramidal neurons and DG granule neurons. a Schematic representation of electrophysiological recording setup for CA1 pyramidal neurons. For panels (b), (c), (e), and (f), open circles are single pairs of control and transfected neurons, filled circles represent the mean amplitudes $( \pm$ SEM), insets show representative current traces from control (black) and transfected (green) neurons with stimulation artifacts removed. Scale bars: 20 ms for AMPA, 50 ms for NMDA, 20 pA. b PSD-95, PSD-93, and SAP102 knockdown significantly decreases AMPAR-eEPSC amplitude in CA1 pyramidal neurons ( $n=8$ pairs, $p=0.00006$, paired $T$ test). c PSD-95, PSD-93, and SAP102 knockdown significantly decreases NMDAR-eEPSC amplitude in CA1 pyramidal neurons ( $n=6$ pairs, $p=0.03$, paired $T$ test). d Schematic representation of electrophysiological recording setup for DG granule neurons. e PSD-95, PSD-93, and SAP102 knockdown significantly decreases AMPAR-eEPSC amplitude in DG granule neurons ( $n=7$ pairs, $p=0.03$, paired $T$ test). f PSD-95, PSD-93, and SAP102 knockdown significantly decreases NMDAR-eEPSC amplitude in DG granule neurons ( $n=6$ pairs, $p=0.045$, paired $T$ test). $\mathbf{g}$, $\mathbf{h}$ Summary bar graphs show the average AMPAR-eEPSC amplitudes ( \pm SEM) (g) and NMDAR-eEPSC amplitudes ( \pm SEM) (h) of CA1 pyramidal neurons and DG granule neurons expressing PSD-95, PSD-93, SAP102 miRs (green) normalized to their respective control cell average eEPSC amplitudes (black). Two-sample $T$ tests were used to compare across independent conditions (CA1 vs. DG); (CA1 vs. DG (AMPA), $n=8$ CA1 pairs, 7 DG pairs, $p=0.12$; CA1 vs. DG (NMDA), $n=6$ CA1 pairs, 6 DG pairs, $p=0.34$ ). ${ }^{\star} p<0.05 ;$ n.s., not significant. All statistical tests performed were two-tailed. Source data are provided in the Source Data file.

triple MAGUK miR construct ${ }^{60}$ through biolistic transfection and stimulating Schaffer collaterals (Fig. 3a) or perforant pathways (Fig. 3d), respectively. Similar to a previous report ${ }^{60}$, we found that knocking down PSD-95, PSD-93, and SAP102 in CA1 pyramidal neurons led to reductions in both AMPAR- (Fig. 3b, g) and NMDAR-eEPSC amplitudes (Fig. 3c, h). When we repeated the experiment in DG granule neurons, we found that the resulting reductions in AMPAR- and NMDAR-mediated current amplitudes were nearly identical to the deficits in CA1 neurons following the same genetic manipulation (Fig. 3e-h).
Our observation of very similar synaptic phenotypes in CA1 and DG granule neurons following knockdown of PSD-95, PSD-93, and SAP102 demonstrates that these MAGUK proteins do not play a unique role in DG granule neurons in general. Rather, these data demonstrate that $\beta$ SAP97 plays a unique and vital synaptic regulatory role specifically in DG granule neurons in a manner that is distinct from other MAGUKs.

Inhibition of $\beta$ SAP97 function in the dentate gyrus disrupts contextual episodic memory. Microdeletion mutations resulting 
in reduced SAP97 expression have been linked to a 40-fold increase in the risk of developing schizophrenia ${ }^{8,9}$. Given that reduced $\beta$ SAP97 expression results in dramatic augmentation of glutamatergic synapse strength in the DG granule neurons, we were interested in whether reduced $\beta S A P 97$ expression within the dentate gyrus produces behavioral phenotypes associated with schizophrenia. A favored cognitive model of the abnormal behaviors and experiences characteristic of schizophrenia suggests that they may be linked to a disturbance in the effects of context ${ }^{41,46-52}$. More specifically, it has been suggested that individuals with schizophrenia exhibit a diminished ability to assess the relative importance of contextual cues in their environment, and that such a deficit may ultimately lead to the development of delusions, disorganization, hallucinations, and the loss of a sense of personal identity ${ }^{40-45}$. A prediction of this model of schizophrenia is that the normal effects of context on memory should be strongly reduced in patients with schizophrenia, as this type of information is believed to be poorly integrated into the episodic representation ${ }^{69}$. Thus, we were interested in whether reduced 3 SAP97 expression specifically in the dentate gyrus produces a deficit in contextual information processing in rodents.

To determine whether diminished BSAP97 expression specifically within the dentate gyrus affects the ability of animals to process contextual information, we performed bilateral stereotaxic injections of our AAV virus containing the $\beta$ SAP97-miR expression construct into either the dentate gyrus or the hippocampal CA1 region of Sprague Dawley rats (Fig. 4a and Supplementary Fig. 4). Following viral transduction, we examined contextual episodic memory in these animals by assessing performance on a Novel Object in Context task ${ }^{70-72}$. In this task, rats were acclimated to a unique combination of objects in two different contexts, Context 1 and Context 2 (Fig. 4b). The animals were then placed in Context 2 containing the combination of objects from Context 1. One of these objects was previously unique to Context 1 and was novel in Context 2. Rats are capable of recognizing this novel, "out of context", object as evidenced by increased exploration time (Fig. 4b). Our results revealed that compared to control animals, $\beta$ SAP97-miR expression in the dentate gyrus led to a significantly reduced time spent exploring the out of context object in Context 2, expressed as a shift from baseline preference for the same object when presented in Context 1 (Fig. 4b; see "methods"). In other words, animals with compromised $\beta$ SAP97 expression in the dentate gyrus did not appear to recognize this object as out of context. In marked contrast, expression of our 3 SAP97-miR in the CA1 region of the hippocampus produced no effect on rat performance on the Novel Object in Context task (Fig. 4b). To ensure that the decreased exploration times of the out of context object we observed with $\beta$ SAP97-miR expression in the dentate gyrus were not secondary to a general avoidance of novel objects due to altered anxiety, we tested anxiety-like behavior using the Zero Maze test. Results showed no differences between groups in time spent in open zones, indicating no effect of dentate gyral $\beta$ SAP97$\mathrm{miR}$ expression on anxiety-related behavior (Fig. 4c). We also observed no effect of dentate gyral 3 SAP97-miR expression on locomotion as indicated by distance traveled in an open field (Fig. 4d). Furthermore, the inability of animals to identify the out of context object following $\beta$ SAP97-miR expression in the dentate gyrus was not due to the inability of these animals to identify novel objects as evidenced by similar performance to controls on a standard perirhinal cortex-dependent Novel Object Recognition task (Fig. 4e). BSAP97-miR expression in the CA1 region of the hippocampus also did not affect performance on the Zero Maze, Open Field or Novel Object Recognition tasks (Fig. 4c-e). Thus, we conclude that compromised $\beta$ SAP97 expression specifically within the dentate gyrus is sufficient to produce a substantial deficit in contextual information processing in rats. These data also demonstrate that disruption of $\beta$ SAP97 function specifically within the dentate gyrus results in behavioral consequences that are related to cognitive impairments observed in individuals with schizophrenia.

Schizophrenia-related mutations in BSAP97 release GluA1containing AMPARs into perforant pathway synapses. Missense mutations in SAP97 have been identified in individuals with schizophrenia and are clustered in SAP97's PDZ2 domain ${ }^{12,14}$ (Fig. 5a). Two unrelated individuals with schizophrenia have the same missense mutation in SAP97, SAP97-G344R ${ }^{12}$ (Fig. 5a). An additional individual with schizophrenia was also identified harboring the de novo mutation SAP97-G357S ${ }^{14}$ (Fig. 5a).

SAP97 binds directly to AMPARs through an interaction between GluA1's seven amino acid C-terminal PDZ-binding domain and SAP97's PDZ2 domain ${ }^{16-20}$ (Fig. 5a). This is in contrast to PSD-95, PSD-93, and SAP102 which associate with AMPAR subunits through an intermediate interaction with transmembrane AMPAR regulatory proteins (TARPs) ${ }^{73}$. The structure of SAP97's PDZ2 domain bound to GluA1's C-terminal PDZ-binding domain has been solved ${ }^{74}$. Using this structure, our protein structural modeling predicted that the schizophreniarelated missense mutations identified in SAP97's PDZ2 domain will inhibit SAP97's ability to associate with GluA1's PDZbinding domain (Fig. 5b and Supplementary Fig. 5). Our modeling was then validated using co-immunoprecipitation of GluA1 with SAP97's PDZ2 domain in HEK293T cells. As predicted, we found that schizophrenia-related missense mutations in SAP97's PDZ2 domain significantly inhibit binding to GluA1 (Fig. 5c and Supplementary Fig. 6a).

Based on our results demonstrating increased AMPAR-eEPSC amplitude following knockdown of $\beta S A P 97$ in DG granule neurons, we postulated that this increase in synaptic AMPAR expression was a result of reduced interaction between 3 SAP97's PDZ2 domain and GluA1's PDZ-binding domain. However, previous studies in CA1 pyramidal neurons have led groups to conclude that GluA1's PDZ-binding domain is dispensable for glutamatergic neurotransmission ${ }^{18,21}$. If disruption of direct interaction between GluA1 and BSAP97 is responsible for the increase in AMPAR-eEPSC amplitude that we observe following knockdown of $\beta$ SAP97 expression in DG granule neurons, then removing GluA1's PDZ-binding domain and preventing its ability to bind to 3 SAP97 should also increase AMPAR-eEPSC amplitude. To test this idea, we employed a molecular replacement approach by biolistically co-transfecting a previously validated GluA1-shRNA construct ${ }^{75}$ and a form of shRNAresistant GluA1 lacking the C-terminal PDZ-binding domain (GluA1- $\Delta 7$ ) (Fig. 5d) in both CA1 and DG granule neurons. Consistent with previous reports ${ }^{18,21}$, we found that molecular replacement of GluA1 with GluA1- $\Delta 7$ in CA1 pyramidal neurons has no effect on AMPAR-eEPSC amplitude (Fig. 5e, g). In marked contrast, we found that molecular replacement of GluA1 with GluA1- $\Delta 7$ in DG granule neurons phenocopied knockdown of $\beta$ SAP97, producing a dramatic increase in AMPAR-eEPSC amplitude (Fig. 5f, g). We also assessed whether replacement of GluA1 with GluA1- $\Delta 7$ occludes a further increase in AMPAReEPSC amplitude produced by $\beta$ SAP97 knockdown in DG granule neurons. We found that further augmentation was indeed occluded (Supplementary Fig. 6b), suggesting that the augmented synaptic strength following GluA1 replacement with GluA1- $\Delta 7$ and following $\beta$ SAP97 knockdown in DG granule neurons results from disruption of the same molecular mechanism. Taken together, these data demonstrate that a direct 
a

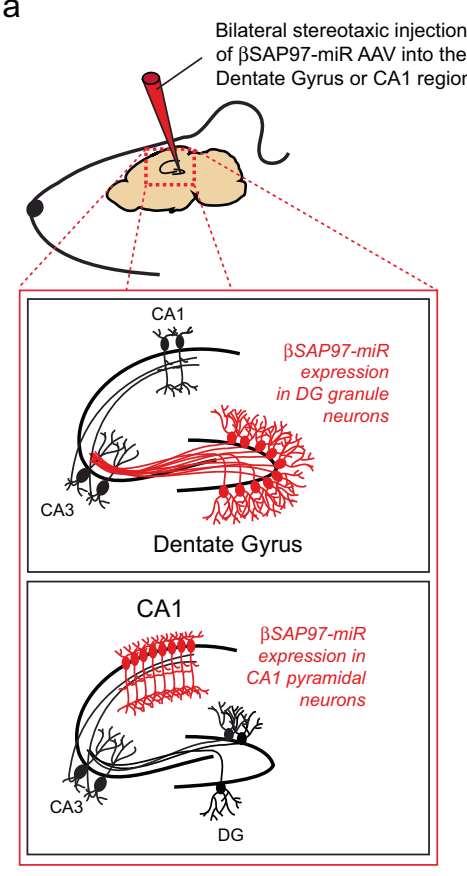

C

Zero Maze b

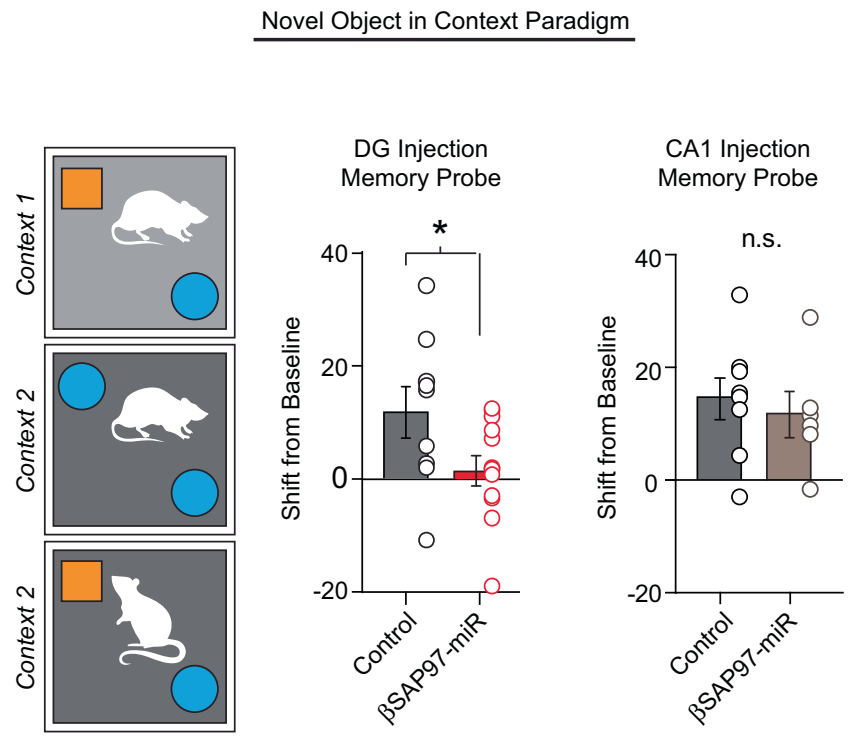

e
Open Field

DG Injection CA1 Injection

DG Injection

CA1 Injection
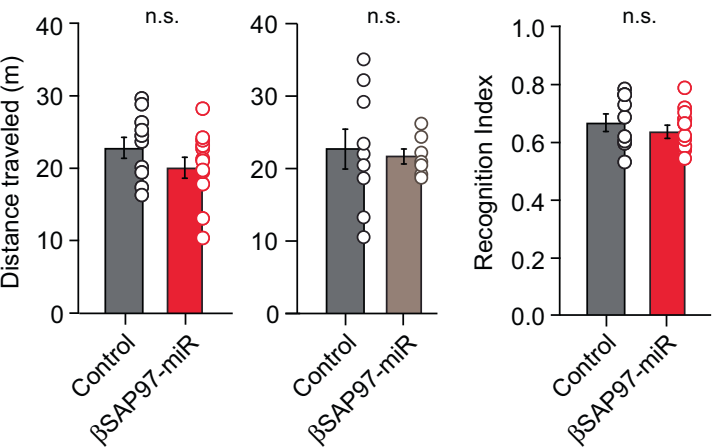

Novel Object Recognition

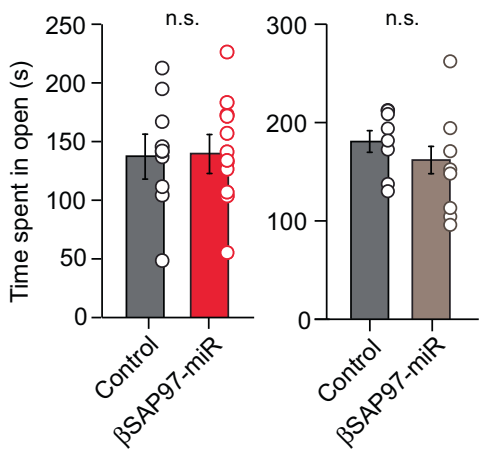

Fig. 4 Knockdown of $\beta$ SAP97 expression in the dentate gyrus disrupts contextual episodic memory. a Illustration of method used to inhibit $\beta$ SAP97 function within the dentate gyrus or the CA1 region of the hippocampus. $\mathbf{b}$ (Left) Experimental setup of the Novel Object in Context behavioral procedure. (Center) Compared to control animals, $\beta S A P 97-m i R$ expression in the dentate gyrus led to a significantly reduced exploration of the object that was novel in Context 2, expressed as a shift from baseline exploration for the same object in Context 1 (control $n=9, \beta S A P 97$ KD $n=12, p=0.04$, ANOVA). (Right) $\beta S A P 97-m i R$ expression in the CA1 region led to no significant changes in exploration of the object that was novel in Context 2 (control $n=8, \beta S A P 97 \mathrm{KD}$ $n=6, p=0.61$, ANOVA). c Control and $\beta$ SAP97 knockdown animals spent similar amounts of time in the open zones in a Zero Maze test for anxiety-like behavior following $\beta$ SAP97-miR AAV injections in either the dentate gyrus (Left, control $n=10, \beta S A P 97 \mathrm{KD} n=12, p=0.91$, two sample $T$ test) or the CA1 region (Right, control $n=8, \beta S A P 97 \mathrm{KD} n=8, p=0.3$, two sample $T$ test). $\mathbf{d}$ Control and $\beta$ SAP97 knockdown animals traveled similar distances in an Open Field test for exploratory behavior and general activity following $\beta$ SAP97-miR AAV injections in either the dentate gyrus (Left, control $n=10$, $\beta$ SAP97 $\mathrm{KD} n=12, p=0.2$, two sample $T$ test) or the CA1 region (Right, control $n=9, \beta S A P 97 \mathrm{KD} n=7, p=0.76$, two sample $T$ test). e Recognition indices of control and $\beta$ SAP97 knockdown animals were similar during a Novel Object Recognition task following $\beta$ SAP97-miR AAV injections in either the dentate gyrus (Left, control $n=9, \beta S A P 97 \mathrm{KD} n=12, p=0.43$, ANOVA) or the CA1 region (Right, control $n=9, \beta S A P 97 \mathrm{KD} n=8, p=0.54$, ANOVA). ${ }^{\star} p<0.05$; n.s., not significant. All statistical tests performed were two-tailed. Source data are provided in the Source Data file.

interaction between GluA1's PDZ-binding domain and $\beta$ SAP97 mediates $\beta$ SAP97's ability to inhibit synaptic AMPAR expression in DG granule neurons. We hypothesized from these data that reducing $\beta$ SAP97 expression releases AMPARs from perisynaptic regions which are in turn captured by traditional MAGUKs at the synapse. If such was the case, knocking down these traditional MAGUK proteins at the synapse would prevent the $\beta$ SAP97 knockdown-mediated augmentation of DG granule neuron synapse function. To test this, we co-expressed the triple
MAGUK miR construct with our $\beta$ SAP97-miR in DG granule neurons, and, consistent with our hypothesis, we found that BSAP97 knockdown does not augment AMPAR-eEPSC amplitude in DG granule neurons on the PSD-95, PSD-93, and SAP102 knockdown background (Supplementary Fig. 6c).

Together, our data suggest that schizophrenia-related missense mutations that inhibit the ability of $\beta S A P 97$ 's PDZ2 domain to interact with GluA1 (Fig. 5c) should produce a potentially pathological release of GluA1-containing AMPARs into the 


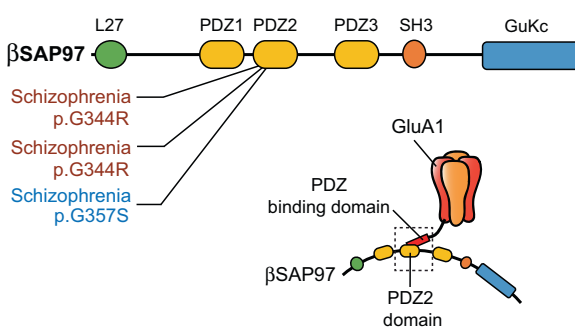

d

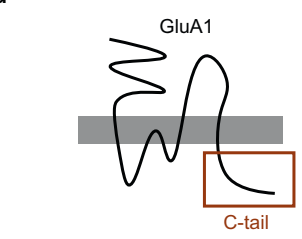

GluA1 C-tail: 884-FPKSMQSIPCMSHSSGM PLGATGL -907 GluA1- $\triangle 7$ C-tail: 884-FPKSMQSIPCMSHSSGM-900

$\mathrm{h}$

Expression of BSAP97-G344R and $\beta$ SAP97-G357S

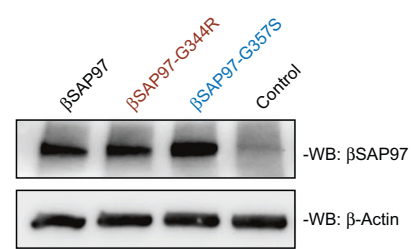

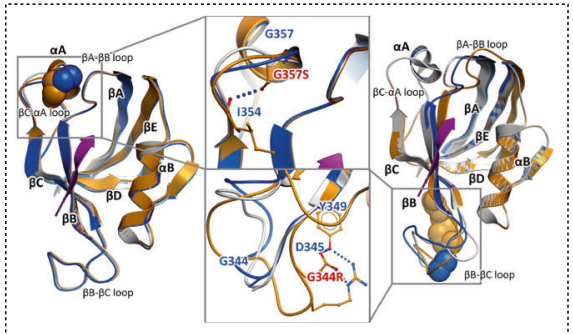

f g

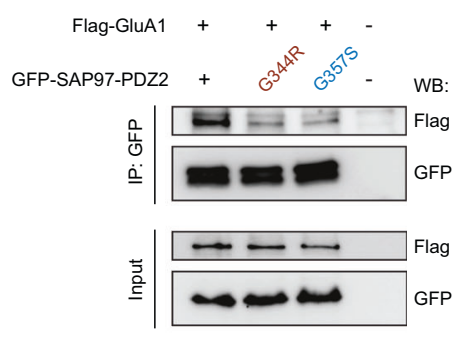

k

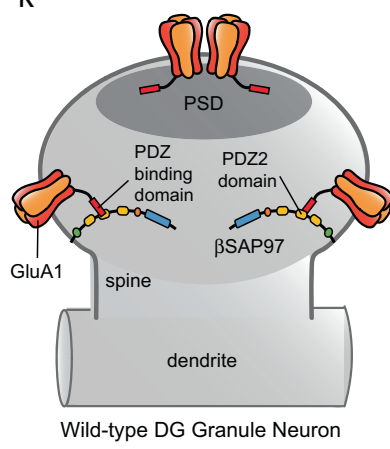

i

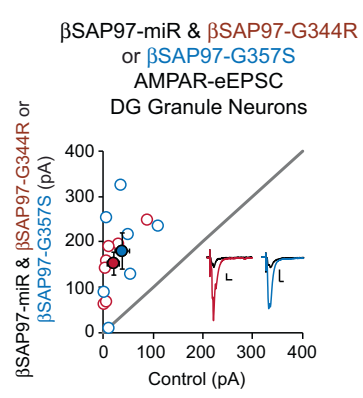

\section{j}

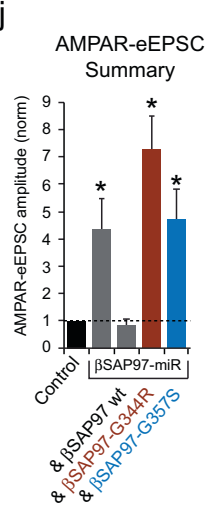

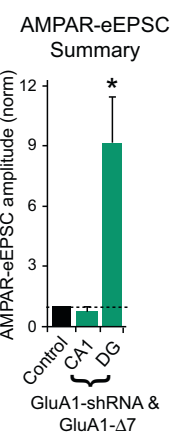
GluA1- $\Delta 7$
Wild-type DG Granule Neuron

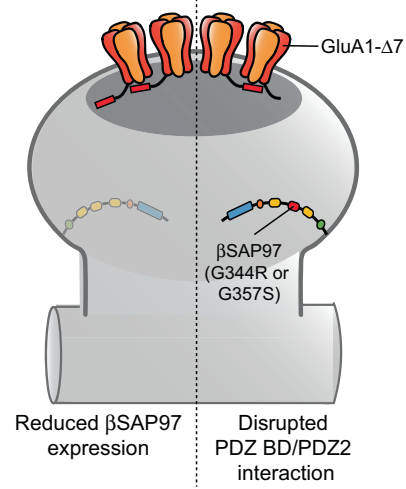

Fig. 5 Clustered schizophrenia-related missense mutations in BSAP97's PDZ2 domain release GluA1-containing AMPARs into perforant pathway synapses. a Illustration of $\beta$ SAP97's protein domain structure and location of schizophrenia-related missense mutations identified in SAP97 (Top). Illustration of interaction between GluA1's PDZ-binding domain and $\beta S A P 97$ 's PDZ2 domain (Bottom, dashed box). b Protein structural modeling of schizophrenia-related mutations in $\beta$ SAP97's PDZ2 domain predict disruption of PDZ2's interaction with GluA1's PDZ-binding domain (see Supplementary Fig. 5 for more details). c Co-immunoprecipitation of FLAG-GluA1 with GFP-SAP97-PDZ2, GFP-SAP97-PDZ2(G344R), or GFP-SAP97-PDZ2(G357S) in HEK293T cells. See methods for details. Quantification for $n=3$ independent experiments provided in Supplementary Fig. 6a. d Topology of the AMPAR subunit GluA1 and C-terminal amino acid sequences for GluA1 and GluA1- $\Delta$ 7. GluA1's PDZ-binding domain (PDZ BD) is highlighted in blue. For panels (e), $(\mathbf{f})$, and (i), open circles in the scatter plots represent single pairs of control and transfected neurons, filled circles represent the mean amplitudes ( \pm SEM), insets show representative current traces from control (black) and transfected (in color) neurons with stimulation artifacts removed. Scale bars: 20 ms, 20 pA. e Molecular replacement by co-expression of the GluA1-shRNA and GluA1- $\Delta 7$ has no effect on AMPAR-eEPSC in CA1 pyramidal neurons $(n=7$ pairs, $p=0.16$, paired $T$ test). $\mathbf{f}$ Molecular replacement by co-expression of GluA1-shRNA and GluA1- $\Delta 7$ significantly increases AMPAR-eEPSC amplitude in DG granule neurons ( $n=7$ pairs, $p=0.016$, paired $T$ test). $\mathbf{g}$ Summary bar graph for $(\mathbf{e})$ and (f) shows the average AMPAR-eEPSC amplitudes ( \pm SEM) of CA1 pyramidal neurons and DG granule neurons with co-expression of GluA1-shRNA and GluA1- $\Delta 7$ normalized to their respective control neurons. $\mathbf{h}$ Representative western blot analysis showing that $\beta$ SAP97-G344R and $\beta$ SAP97-G357S exhibit levels of expression in HEK293 cells similar to that of wildtype $\beta$ SAP97 ( $n=2$ independent experiments). i Molecular replacement of $\beta$ SAP97 with $\beta$ SAP97-G344R and $\beta$ SAP97-G357S in DG granule neurons significantly increases AMPAR-eEPSC amplitude ( $\beta$ SAP97-G344R, $n=7$ pairs, $p=0.0004$, paired $T$ test; $\beta$ SAP97-G357S, $n=7$ pairs, $p=0.01$, paired $T$ test). $\mathbf{j}$ Summary bar graph shows the average AMPAR-eEPSC amplitudes ( \pm SEM) of the conditions compared in panel (i). ${ }^{*} p<0.05$. $\mathbf{k}$ Model of $\beta S A P 97-$ mediated regulation of glutamatergic synaptic function. GluA1-containing AMPARs on the surface of neurons are held perisynaptically by an interaction between $\beta$ SAP97 and GluA1's PDZ-binding domain. Reducing $\beta$ SAP97 expression or inhibiting GluA1's ability to bind to $\beta$ SAP97's PDZ2 domain results in local rearrangement of AMPA receptor organization in spines whereby AMPA receptors normally held by $\beta$ SAP97 perisynaptically are released and then grabbed by PSD-95, PSD-93, and SAP102 in the PSD. All statistical tests performed were two-sided. Source data are provided in the Source Data file. 
glutamatergic synapses of DG granule neurons. Before directly testing whether schizophrenia-related missense mutations in $\beta S A P 97$ 's PDZ2 domain influence synaptic AMPAR function, we first examined the expression of wild-type $\beta S A P 97, \beta S A P 97-$ G344R, and $\beta$ SAP97-G357S. We observed levels of $\beta$ SAP97G344R and $\beta$ SAP97-G357S expression that were comparable to wild-type $\beta$ SAP97 (Fig. 5h). Additionally, we observed targeting of $\beta$ SAP97-G344R and $\beta$ SAP97-G357S to dendritic spines in DG granule neurons like that observed with wild-type $\beta S A P 97$ (Supplementary Fig. 6d, 2d). We then examined AMPAReEPSC amplitude in DG granule neurons where endogenous BSAP97 was molecularly replaced with $\beta$ SAP97-G344R or $\beta$ SAP97-G357S (Fig. 5a, i, j). In contrast to molecular replacement with wild-type $\beta$ SAP97 (Figs. 1d, 5j), we found that both schizophrenia-related mutant forms of $\beta$ SAP97 produced dramatic increases in synaptic AMPAR-eEPSC amplitude in DG granule neurons like that seen with knockdown of $\beta$ SAP97 and molecular replacement of GluA1 with GluA1- $\Delta 7$ (Fig. 5i, j). Molecularly replacing $\beta$ SAP97 with $\beta$ SAP97-G344R, which produced the largest increase in AMPAR-eEPSC amplitude of the two missense mutations, was found to have no effect on dendritic AMPAR surface current (Supplementary Fig. 6e). As with reduced $\beta S A P 97$ expression, such data demonstrate that the AMPAR-eEPSC augmentation produced by these schizophreniarelated missense mutations results from rearrangement of AMPARs on the dendritic surface of DG granule neurons. Finally, we performed quantal analysis on our $\beta$ SAP97 knockdown AMPAR-eEPSC data and on our AMPAR-eEPSC data from molecular replacements with the schizophrenia-related mutants to determine whether the augmented AMPAR-eEPSC amplitude in these conditions is caused by an increase in quantal size or quantal content $54,55,57,60,76,77$. We found that the augmentations in all three conditions were caused by an increase in quantal size rather than quantal content (Supplementary Fig. 6f). Thus, the increases in AMPAR-eEPSC amplitude we observe are due to increased AMPAR expression at existing functional glutamatergic synapses rather than an increase in glutamatergic synapse number.

Altogether, our data demonstrate that both reduced $\beta$ SAP97 expression and schizophrenia-related missense mutations in $\beta S A P 97$ 's PDZ2 domain compromise $\beta$ SAP97's interaction with GluA1-containing AMPARs and, as a result, cause rearrangements of AMPARs present on the dendritic surface of DG granule neurons that lead to increased AMPAR expression in the PSDs of existing functional glutamatergic synapses. We observe robust localization of $\beta$ SAP97 in the spines of DG granule neurons that is consistent with previous work demonstrating a perisynaptic localization of $\beta S A P 97^{61}$. Therefore, we believe the most likely explanation for our findings is that $\beta S A P 97$ maintains perisynaptic pools of AMPARs in DG granule neurons (Fig. 5k), and that inhibiting 3 SAP97's interaction with GluA1 by way of reduced $\beta$ SAP97 expression or missense mutations in $\beta$ SAP97's PDZ2 domain causes AMPARs to translocate from these perisynaptic regions to the PSD resulting in aberrant increases in perforant pathway synapse strength (Fig. 5k). Such elevations in the strength of perforant pathway synapses likely disrupt dentate gyral information processing and may contribute to the development of behavioral phenotypes associated with schizophrenia.

\section{Discussion}

While the synaptic roles of most MAGUK proteins are well understood (i.e. PSD-95, PSD-93, and SAP102), the role of SAP97 in regulating glutamatergic neurotransmission has remained unclear. In previous studies, overexpression of recombinant
SAP97 in neurons has led to mixed and inconclusive results ${ }^{22-27}$. It is difficult to derive meaningful conclusions from such experiments given inconsistencies in the SAP97 splice variants used across studies. Furthermore, overexpressing recombinant proteins in neurons that do not utilize the protein endogenously may lead to aberrant protein function. Inhibiting SAP97 expression has also been shown to produce a variety of seemingly contradictory glutamatergic synapse phenotypes $22,25,28$. Such discrepancies have likely arisen at least in part because of the different neuronal preparations used. In the case of dissociated neuron cultures, neurons lose specificity in their synaptic connections and instead form synapses with neighboring neurons regardless of the original circuitry. In such artificial contexts, neurons may develop synapses utilizing proteins they otherwise would not. Critical controls for SAP97 RNAi usage, such as rescue experiments, are also missing in previous studies making it difficult to rule out off-target effects. Thus, previous literature has led to considerable confusion regarding SAP97's role in synaptic regulation and, based on the absence of a synaptic phenotype in CA1 pyramidal neurons following knock out of SAP97 ${ }^{28}$, groups have generally concluded that SAP97 has no role in the regulation of glutamatergic synapse function ${ }^{15}$.

Driven by the implication of dentate gyral and SAP97 dysfunction in schizophrenia, the present study visually identified the perforant pathway-DG granule neuron synapse as where SAP97 might play an important synaptic role due to the robust endogenous $\beta$ SAP97 expression in the dendrites of DG granule neurons. We determined that inhibiting $\beta$ SAP97 function specifically in DG granule neurons leads to a dramatic increase in AMPARmediated currents, signaling an increase in synaptic AMPAR expression. In contrast, we observed no effects on synaptic transmission following knockdown of $\beta$ SAP97 in CA1 pyramidal neurons, as predicted from the lack of dendritic expression of BSAP97 in CA1 neurons. Taken together, our data demonstrate that BSAP97 plays a critical cell-type-specific regulatory role at perforant pathway-DG granule neuron synapses. Thus, we have identified a specific set of synapses where $\beta$ SAP97 is essential for regulating glutamatergic neurotransmission. In contrast to a previous study performed in dissociated hippocampal neurons ${ }^{25}$, we find that $\beta$ SAP97 does not play a significant role in governing synaptic NMDAR function. The lack of an effect we observe on NMDAR function is consistent with previous biochemical evidence suggesting that SAP97 does not interact with NMDARs ${ }^{16,78}$. One possible explanation for this discrepancy is that the formation of glutamatergic synapses between hippocampal neurons outside of their native circuitry confers unique properties to $\beta$ SAP97. Intriguingly, we also observe a laminar pattern of $\beta$ SAP97 immunolabeling within the molecular layer of the dentate gyrus that is consistent with higher levels of $\beta$ SAP97 expression in the synapses of the lateral perforant pathway relative to the medial perforant pathway. Going forward, it will be interesting to determine if $\beta$ SAP97 inhibition of AMPARmediated synaptic transmission is greater at lateral perforant pathway synapses compared to those of the medial perforant pathway.

Having identified the synapses where SAP97 plays a critical role, we began characterizing the mechanism by which $\beta$ SAP97 regulates synaptic AMPAR expression. We found through IV rectification analysis that 3 SAP97 appears not to govern synaptic AMPAR subunit composition. GABAergic neurotransmission and intrinsic excitability were also unaffected by $\beta$ SAP97 knockdown in DG granule neurons. Furthermore, we determined through exogenous glutamate application that while the synaptic AMPAR function increased following $\beta$ SAP97 knockdown, AMPAR expression on the surface of DG granule neuron dendrites did not change. This demonstrates that the increase in 
synaptic AMPAR function we observe is not due to the exocytosis of intracellular AMPARs but rather a reorganization of AMPARs on the dendritic surface (Fig. 5k). Additionally, we found that knocking down MAGUKs PSD-95, PSD-93, and SAP102 decreased synaptic transmission similarly in CA1 pyramidal neurons and DG granule neurons. This observation ruled out the possibility that the dramatic increase in synaptic AMPAR expression following 3 SAP97 knockdown in DG granule neurons was due to all MAGUK proteins playing a fundamentally different role in the dentate gyrus. Thus, our data demonstrate that BSAP97 is distinct from the other MAGUKs in its function.

In humans, microdeletion mutations involving the DLG1 gene are believed to lower BSAP97 expression and contribute to a greater than 40 -fold increase in the risk of developing schizophrenia ${ }^{8,9}$. The development of schizophrenia-related disorders has also been linked to disruption of dentate gyral information processing ${ }^{1,30-38}$. Given that reduction of $\beta S A P 97$ expression resulted in dramatic augmentation of glutamatergic synapse strength in DG granule neurons, we were interested in whether reducing BSAP97 expression specifically within the dentate gyrus is sufficient to produce behavioral phenotypes associated with schizophrenia. A well-established model of schizophrenia suggests that deficits in processing contextual information during episodic memory formation may underlie core phenotypes associated with schizophrenia including delusions, disorganization, hallucinations, and the loss of a sense of personal identity ${ }^{40-45}$. Here, we investigated contextual episodic memory formation in rats following the expression of our $\beta$ SAP97-miR in the dentate gyrus or the CA1 region of the hippocampus. We found that animals with $\beta$ SAP97-miR expression in the dentate gyrus exhibited substantial impairment in contextual information processing during episodic memory formation. In marked contrast, $\beta$ SAP97-miR expression in the CA1 region of the hippocampus produced no effect on contextual information processing. Thus, contextual information processing is only compromised by our $\beta$ SAP97-miR in the brain region where we observe dendritic expression of $\beta$ SAP97 and where glutamatergic synapse function is augmented when $\beta$ SAP97 expression is knocked down. Together, our results establish that disruption of $\beta$ SAP97 function specifically within the dentate gyrus is sufficient to produce behavioral deficits consistent with those observed in individuals with schizophrenia. Given that we see no change in GABAergic neurotransmission or intrinsic excitability in DG granule neurons following knockdown of $\beta$ SAP97 expression, we believe that the augmented glutamatergic synapse strength we observe in these neurons is likely responsible for the deficit in contextual information processing in these animals. However, we do acknowledge that we cannot definitively exclude the relevance of some yet-tobe-discovered role of $\beta S A P 97$ in regulating DG neuron function.

Traditional MAGUK proteins (PSD-95, PSD-93, and SAP102) interact with AMPARs through TARP proteins, which contain PDZ-binding motifs ${ }^{73}$. $\beta S A P 97$ differs from these MAGUKs in that its PDZ2 domain can directly interact with the C-terminal PDZ-binding domain of the AMPAR subunit GluA1 ${ }^{16-20}$. In humans, schizophrenia-related missense mutations in SAP97 are clustered in SAP97's PDZ2 domain and here we show that these mutations inhibit GluA1 binding to SAP97's PDZ2 domain. These data suggested that inhibition of SAP97's interaction with GluA1 may be responsible for the increased synaptic AMPAR function we observe in DG granule neurons, which may, in turn, contribute to the development of schizophrenia in humans. In contrast to traditional MAGUK proteins, BSAP97 possesses an $\mathrm{N}$-terminal L27 domain that causes $\beta$ SAP97 to localize to perisynaptic regions in spines outside of the PSD ${ }^{61,79}$. Based on this previous work and our own 3 SAP97 localization data, we hypothesized that endogenous $\beta$ SAP97 may sequester GluA1- containing AMPARs perisynaptically in DG granule neurons through a direct interaction between $\beta$ SAP97 and GluA1 subunits. Reduced $\beta$ SAP97 expression or schizophreniarelated missense mutations in $\beta$ SAP97's PDZ2 domain may disrupt the direct interaction between $\beta S A P 97$ and GluA1's C-terminal PDZ-binding domain causing AMPARs to be released into the PSD. If this is the case, removing GluA1's PDZ-binding domain in DG granule neurons should result in elevated synaptic AMPAR function like that produced by $\beta$ SAP97 knockdown. Removal of GluA1's PDZ-binding domain (GluA1- $\Delta 7$ ) was previously examined in CA1 pyramidal neurons and produced no effect on glutamatergic neurotransmission, suggesting that in these neurons a GluA1- 3 SAP97 interaction likely does not play a regulatory role ${ }^{18}$. In fact, the relevance of GluAl's entire C-tail in the regulation of glutamatergic neurotransmission was recently called into question ${ }^{21}$. In agreement with these studies, we found that molecularly replacing endogenous GluA1 with GluA1- $\Delta 7$ in CA1 pyramidal neurons produced no effect on baseline glutamatergic neurotransmission. However, when repeating the experiment in DG granule neurons, we found a dramatic increase in AMPAR-eEPSC amplitude that phenocopies what is observed with knocking down $\beta$ SAP97. We also found that GluA1- $\Delta 7$ molecular replacement in DG granule neurons prevents further augmentation of synaptic strength by our $\beta$ SAP97-miR. Together, such data demonstrate that disruption of $\beta$ SAP97's interaction with GluA1's C-terminal PDZ-binding domain produces the dramatic increases in AMPAR-eEPSC amplitude that we observe in DG granule neurons (Fig. 5k).

Our protein structural modeling and co-immunoprecipitation data demonstrate that schizophrenia-related mutations clustered in BSAP97's PDZ2 domain inhibit GluA1's ability to bind to this domain. Such data led us to predict that these mutant forms of BSAP97 would produce pathological augmentation of synaptic AMPAR function in DG granule neurons. Indeed, we find that molecular replacement of $\beta$ SAP97 with these schizophreniarelated mutant forms of $\beta$ SAP97 produce dramatic augmentation of AMPAR-eEPSC amplitude in DG granule neurons like that seen with $\beta$ SAP97 knockdown and molecular replacement of GluA1 with GluA1- $\Delta 7$. Quantal analysis performed on these data demonstrate that the increase in AMPAR-eEPSC amplitude that we observe with these mutations is due to increased AMPAR expression at existing functional glutamatergic synapses. This finding is in line with previous work showing that recombinant BSAP97 accumulates in dendritic spines as they structurally mature ${ }^{80}$. We also show that these mutations do not impact BSAP97 expression, targeting of $\beta$ SAP97 to spines, or dendritic AMPAR surface current. Altogether, our data suggest that BSAP97 binds to the C-terminal PDZ domain of GluA1containing AMPARs and, through this interaction, maintains a perisynaptic surface pool AMPARs within the spines of DG granule neurons (Fig. 5k). This mechanism is consistent with that proposed by a previous study performed utilizing recombinant BSAP97 in neurons ${ }^{61}$. Furthermore, our data suggest that when the interaction between $\beta$ SAP97 and GluA1 is disrupted, either by reducing $\beta$ SAP97 expression or schizophrenia-related missense mutations in BSAP97's PDZ2 domain, AMPARs are released from perisynaptic sites into the PSD causing a pathological increase in synaptic strength in DG granule neurons (Fig. 5k) that likely disrupts information processing in the dentate gyrus. Going forward, it will be important to determine why DG granule neurons uniquely employ $\beta S A P 97$ to sequester AMPARs outside of the PSD.

In conclusion, our study identifies a cell-type-specific synaptic regulatory mechanism in the dentate gyrus that, when disrupted, impairs contextual information processing in rats. As a result, restoring proper $\beta$ SAP97 function and/or reducing AMPAR- 
mediated neurotransmission within the dentate gyrus should be considered when designing potential therapeutic strategies for individuals harboring pathological mutations in the DLG1 gene. In future studies, it will also be important to determine whether similar synaptic pathology in the dentate gyrus is produced by schizophrenia-related mutations in other genes. Information derived from such studies will be instrumental in establishing how broadly applicable these therapeutic strategies might be in the treatment of this psychiatric disorder.

\section{Methods}

Experimental constructs. $\beta$ SAP97-miR target sequence $5^{\prime}$-TCTACTGGAGGGCTAAGGCCG-3' ${ }^{\prime}$ was embedded into an emerald GFP (emGFP) sequence in a pFUGW expression vector to make a pFUGW- 3 SAP97-miR construct. For the SAP97 rescue experiment, cDNA for RNAi-resistant human $\beta$ SAP97 with inserts I1B, I3, and I5 was obtained from GE Dharmacon (CloneId: 9053182) and cloned into NheI and XmaI sites of a pCAGGS-IRES-mCherry expression vector. Schizophrenia-related missense mutants $\beta$ SAP97-G344R and $\beta$ SAP97-G357S were made from this pCAGGS- $\beta$ SAP97-IRES-mCherry construct using overlapextension PCR followed by In-Fusion cloning (Clontech). mCherry-tagged $\beta$ SAP97 constructs (wild-type, G344R and G357S) were made by deleting the IRES element and fusing the mCherry onto the C-terminus of $\beta$ SAP97 in the pCAGGS- $\beta$ SAP97IRES-mCherry and mutant constructs. The chained, triple MAGUK miR construct was previously validated ${ }^{60}$ and was generously provided by Dr. Roger Nicoll. Rat GluA1 (flip-type) construct pCAGGS-GluA1-IRES-GFP, pCAGGS-FLAG-GluA1, and the FHUGW-GluA1shRNA construct (target sequence 5' - GGAATCCGAAAGATTGGTT - 3') were generously provided by Dr. Katherine Roche ${ }^{75}$. An RNAi-resistant form of GluA1 was generated by introducing five silent mutations within the shRNA target sequence via overlap-extension PCR followed by InFusion cloning (Clontech). shRNA-resistant GluA1 missing the C-terminal PDZbinding domain (GluA1- $\Delta 7$ ), specifically missing the last seven amino acid residues PLGATGL, was generated also using the overlap-extension PCR and In-Fusion methods. All plasmids were confirmed by DNA sequencing. Oligonucleotide sequences used to generate experimental constructs are provided in Supplementary Table 1.

Immunohistochemistry. Experiments were performed in accordance with NIH Guidelines for the Care and Use of Laboratory Animals, and all procedures were approved by the Institutional Animal Care and Use Committee of the University of Southern California. Postnatal day 15 (P15) Sprague Dawley rats of both sexes were transcardially perfused with $11 \mathrm{ml}$ of cold PBS and $25 \mathrm{ml}$ of cold $4 \%$ PFA in PBS at a flow rate of $3 \mathrm{ml} / \mathrm{min}$. The hippocampi were immediately dissected and were post-fixed overnight at $4{ }^{\circ} \mathrm{C}$ in $4 \%$ PFA. After 3 brief washes in PBS, the hippocampi were sliced using a vibratome at $100 \mu \mathrm{m}$ thickness. Slices were placed into $24-$ well culture plates containing PBS and stained within the wells. Slices were blocked in PBST (PBS $+0.25 \%$ TritonX-100) with $10 \%$ Goat Serum for $1 \mathrm{~h}$ at room temperature, rinsed in PBST, and incubated with primary antibody diluted in PBST overnight at $4{ }^{\circ} \mathrm{C}$. Then the slices were thoroughly washed in PBST and stained with secondary antibody diluted in PBST for $2 \mathrm{~h}$ at room temperature. Slices were then mounted onto slides, dried for $15 \mathrm{~min}$, and mounted with either

Fluoromount-G (SouthernBiotech, Cat\#0100-01) or Fluoroshield with DAPI (Sigma Aldrich, Cat\#F6057). For the immunizing peptide block experiment, a blocking peptide matching the epitope sequence of the rabbit anti-SAP97 antibody EEYRSKLSQTEDRQLRSS was synthesized ( $>98 \%$ purity). Diluted SAP97 primary antibody was prepared as normal, the blocking peptide was added to the antibody at a 10:1 ratio, and the mixture was incubated overnight at $4{ }^{\circ} \mathrm{C}$. This peptideblocked antibody was used following the same staining protocol as detailed above. Antibodies used are as follows: rabbit anti-SAP97 (1:1000, Invitrogen Cat\#PA1044, RRID: AB_2092021), mouse anti-MAP2 (1:1000, Sigma Aldrich Cat\#4403), rabbit IgG polyclonal isotype control antibody (1:1000, Abcam Cat\#171870), rabbit anti-SAP97 (100ug, custom made, YenZym Antibodies, epitope sequence: DQSEQETSDADQ), goat anti-rabbit Alexa Fluor 555 (1:1000, Invitrogen Cat\#A32732, RRID: AB_2633281), goat anti-mouse Alexa Fluor 488 (1:1000, Invitrogen Cat\#A-11001, RRID: AB_2534069). Slides were imaged with a Keyence All-in-One Fluorescence Microscope BZ-X800 with a 4x objective for whole hippocampal slice imaging and with Zeiss 880 Confocal Microscope with 10x and 40x water-immersion objectives.

AAV production. Our emGFP- $\beta S A P 97-m i R$ expression construct was subcloned into AAV-bGH(+) and packaged into an adeno-associated virus (AAV2; Vector Biolabs) under the control of a UbC promoter to create the 3 SAP97 miR AAV (titer $=1.0 \mathrm{e} 13 \mathrm{GC} / \mathrm{ml}$ ). A scrambled miR, emGFP-expressing AAV2 downstream of a UbC promoter (titer $=2.1 \mathrm{e} 13 \mathrm{GC} / \mathrm{ml}$ ) was used as a control (Vector Biolabs).

Immunoblotting and Co-immunoprecipitation. For knockdown experiments in primary rat hippocampal dissociated neurons, neurons were prepared from E18.5 Sprague Dawley rats (Charles River Laboratories, Wilmington, MA, USA) of both sexes and transduced with either $\beta$ SAP97-miR AAV or scrambled-miR AAV at DIV1. Lysates were prepared at DIV14 in RIPA buffer containing protease inhibitor mix (ThermoFisher, Halt Protease Inhibitor Cocktail). Proteins were resolved by SDS-PAGE. Following the transfer, membranes were cut and analyzed by western blot with antibodies against SAP97 (1:1000, Invitrogen, Cat\#PA1-741, RRID: AB_2092020), PSD-95 (1:1000, Millipore, Cat\#637258), PSD-93 (1:1000, Millipore, Cat\#618436), SAP102 (1:1000, Biolegend, Cat\#832004), and $\beta$-actin (1:1000, Cell Signaling Technology, Cat\#4970S). Goat anti-rabbit, HRP-linked secondary antibody (1:10000, Cell Signaling Technology, Cat\#7074S) or horse antimouse, HRP-linked secondary antibody (1:10000, Cell Signaling Technology, Cat\#7076S) were used for all immunoblotting experiments described. For validating $\beta$ SAP97 rescue by the miR-resistant BSAP97 construct, HEK293 cells (ATCC, Cat\#CRL-1573) cultured in DMEM supplemented with $10 \%$ FBS and $1 \%$ penicillin-streptomycin and maintained at $37^{\circ} \mathrm{C}$ and $5 \% \mathrm{CO} 2$ were transfected using Lipofectamine 2000 (Invitrogen, Cat\#11668027) with the following con-

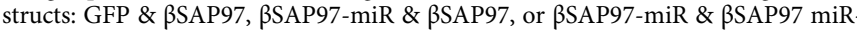
resistant. Cells were harvested $72 \mathrm{~h}$ post-transfection. Lysates were prepared in RIPA buffer containing protease inhibitor mix, and proteins were resolved by SDSPAGE. Following the transfer, membranes were cut and analyzed by western blot with antibodies against SAP97 (1:500, Neuromab, Cat\#75-030) and $\beta$-actin (1:1000 Cell Signaling Technology, Cat\#4970S). For schizophrenia mutant expression experiments, HEK293 cells were transfected with cDNAs of $\beta$ SAP97, $\beta$ SAP97G344R, or $\beta$ SAP97-G357S using Lipofectamine 2000 and harvested 72 h posttransfection. Lysates were prepared in RIPA buffer containing protease inhibitor mix, and proteins were resolved by SDS-PAGE. Following the transfer, membranes were cut and analyzed by western blot with the same SAP97 and $\beta$-actin antibodies and concentrations as the experiments described above.

For co-immunoprecipitation experiments, HEK293T cells (ATCC, Cat\#CRL3216) were co-transfected with FLAG-GluA1 and wild-type GFP-SAP97-PDZ2, GFP-SAP97-PDZ2(G344R), or GFP-SAP97-PDZ2(G357S) using Lipofectamine 2000. $24 \mathrm{~h}$ following transfection, cells were washed and lysed (lysis buffer: $25 \mathrm{mM}$ Tris- $\mathrm{HCl} \mathrm{pH}$ 7.4, $150 \mathrm{mM} \mathrm{NaCl}, 1 \mathrm{mM}$ EDTA, 1\% NP-40, 5\% glycerol). Lysates were rocked for $30 \mathrm{~min}$ at $4{ }^{\circ} \mathrm{C}$ and centrifuged. Supernatants were collected and incubated with anti-GFP antibody (mouse, Neuromab, Cat\#75-131) overnight at $4{ }^{\circ} \mathrm{C}$. Protein G Dynabeads (ThermoFisher, Cat\#10007D) were added to the lysate/ antibody mixture and incubated at $4{ }^{\circ} \mathrm{C}$ for $4 \mathrm{~h}$. Beads were washed and eluted in the Dynabead elution buffer at room temperature to ensure that FLAG-GluA1 was detectable at the expected monomeric molecular weight. Whole-cell lysates used as inputs were boiled to improve resolution and band signal for both FLAG-GluA1 and GFP-SAP97-PDZ2. Proteins were resolved by SDS-PAGE. Following the transfer, membranes were cut and analyzed by western blot with antibodies against FLAG (1:2000, Sigma Aldrich, Cat\#8592) and GFP (rabbit, 1:1000, Invitrogen, Cat\#A-11122). Blots were quantified using Fiji. To compare the interactions between FLAG-GluA1 and WT, G344R, or G357S GFP-SAP97-PDZ2, bands representing immunoprecipitated GFP-SAP97-PDZ2, co-immunoprecipitated FLAG-GluA1, and input FLAG-GluA1 were measured. The fraction of input FLAG-GluA1 that was co-immunoprecipitated was calculated by dividing the coimmunoprecipitated FLAG-GluA1 by input FLAG-GluA1. This number was normalized to immunoprecipitated GFP-SAP97-PDZ WT, G344R, or G357S, resulting in the final numbers used to compare WT, G344R, and G357S GFPSAP97-PDZ2, respectively. All uncropped blots with molecular weight ladders are provided in the Source Data.

Real-time polymerase chain reaction. Rat hippocampal neurons were prepared from E18.5 Sprague Dawley rats (Charles River Laboratories, Wilmington, MA, USA) of both sexes and transduced with either 3 SAP97-miR AAV or scrambled miR AAV at DIV1. At DIV14 cells were suspended in a lysis buffer containing $1 \%$ beta-mercaptoethanol and disrupted using QIAshredder homogenizers (Qiagen, Cat\#79654). Total RNA was purified and isolated with the RNeasy Micro kit (Qiagen, Cat\#74004) following the manufacturer's instructions. Total RNA content was quantified using the Nanodrop One spectrophotometer (ThermoFisher, Cat\#ND-ONE-W) and the Quantitect Reverse Transcription kit was employed to synthesize complimentary DNA from 500 ng of total RNA (Qiagen, Cat\#205311). Real-time polymerase chain reaction (RT-PCR) was run on a QuantStudio 5 RTPCR system (Applied Biosystems, Cat\#A28140) using the Taqman Fast Advanced Master Mix (Applied Biosystems, Cat\#4444557) and Taqman Gene Expression Assay Mix for GAPDH (Assay ID Rn01775763_g1), aSAP97 (Assay ID ART2CT4) and BSAP97 (Assay ID Rn01439452_m1). CT values were obtained from the QuantStudio 5 Design \& Analysis software and converted to fold changes using the Delta-Delta CT method.

Electrophysiology. $400 \mu \mathrm{m}$ rat organotypic entorhino-hippocampal slice cultures were prepared from both male and female P6 to P8 Sprague Dawley rats as previously described ${ }^{81-83}$. Hippocampi with accompanying entorhinal cortices were removed from 6 to 10 rats at a time, and $400 \mu \mathrm{m}$ transverse sections were made using a MX-TS tissue slicer (Siskiyou). Slices from these 6-10 rats were mixed together and then mounted on individual squares of Biopore Membrane filter roll (Millipore) and placed on Millicell Cell Culture inserts (Millipore) in $35 \mathrm{~mm}$ dishes containing $1 \mathrm{ml}$ of culture media (MEM + HEPES (Gibco Cat\#12360-038), horse serum $25 \%$, HBSS $(25 \%)$ and L-glutamine $(1 \mathrm{mM})$. Media was exchanged every 
other day. Slices with large portions of entorhinal cortex were visually identified subsequent to slicing. These slices were selected and plated for use in our experiments, and the presence of entorhinal cortex was again confirmed when selecting slices appropriate for data acquisition. Culture media was exchanged every other day. Sparse biolistic transfections were performed on DIV1 as described in detail previously $^{23}$. 50ug total of mixed plasmid DNA was coated on $1 \mu \mathrm{m}$-diameter gold particles in $0.5 \mathrm{mM}$ spermidine, precipitated with $0.1 \mathrm{mM} \mathrm{CaCl}_{2}$, and washed four times in pure ethanol. These DNA-coated gold particles were then coated onto PVC tubing, dried using ultra-pure $\mathrm{N}_{2}$ gas, and stored at $4{ }^{\circ} \mathrm{C}$ in desiccant. Before use, the gold particles were brought up to room temperature and then delivered to slice cultures via a Helios Gene Gun (BioRad). For biolistically transfecting more than one construct, equal amounts of plasmid DNA for each construct was used. Construct expression was confirmed by GFP or mCherry epifluorescence. Electrophysiological recordings were performed on DIV7 slices. During recordings, slices were maintained in room-temperature artificial cerebrospinal fluid (aCSF) external solution containing (in mM): $119 \mathrm{NaCl}, 2.5 \mathrm{KCl}, 1 \mathrm{NaH}_{2} \mathrm{PO}_{4}, 26.2$ $\mathrm{NaHCO}_{3}, 11$ glucose, $4 \mathrm{CaCl}_{2}$, and $4 \mathrm{MgSO}_{4} .5 \mu \mathrm{M}$ 2-chloroadenosine and $0.1 \mathrm{mM}$ picrotoxin were also added to the aCSF to dampen epileptiform activity and block $\mathrm{GABA}_{\mathrm{A}}$ receptor activity, respectively. Osmolarity was adjusted to $310-315 \mathrm{mOsm}$. aCSF was saturated with $95 \% \mathrm{O}_{2} / 5 \% \mathrm{CO}_{2}$ throughout the recording. Borosilicate recording electrodes were filled with an internal, whole-cell recording solution containing (in mM): $135 \mathrm{CsMeSO}_{4}, 8 \mathrm{NaCl}, 10$ HEPES, 0.3 EGTA, 5 QX-314, 4 Mg-ATP, and 0.3 Na-GTP. The internal solution was adjusted to pH 7.3-7.4 and osmolarity of 290-295 mOsm.

Transfected DG granule neurons and CA1 pyramidal neurons were identified using epifluoresence microscopy. Dual whole-cell recordings of either neuronal subtype were made through simultaneous recordings from one transfected neuron and a neighboring non-transfected control neuron. Postsynaptic responses were evoked by stimulating with a monopolar glass electrode filled with aCSF placed in the middle of the molecular layer to stimulate both medial and lateral perforant pathway afferents for DG granule neuron recordings and at the stratum radiatum/ stratum lacunosum-moleculare border or in the stratum oriens for CA1 pyramidal neuron recordings. The responses were acquired using a Multiclamp 700B amplifier (Molecular Devices), filtered at $2 \mathrm{kHz}$, and digitized at $10 \mathrm{kHz}$. AMPAR evoked EPSCS (eEPSCs) were measured at $-70 \mathrm{mV}$. NMDAR-eEPSCs were measured at $+40 \mathrm{mV}$ and were temporally isolated by measuring amplitudes $150 \mathrm{~ms}$ following the stimulus, at which point the AMPAR-eEPSC had completely decayed. Data analysis was performed using Igor Pro (Wavemetrics). In the scatter plots for simultaneous dual whole-cell recordings, each open circle represents one paired recording, and the closed circle represents the average of all paired recordings. The diagonal line is shown on the scatter plot to demonstrate that if the data point falls above the diagonal line, it indicates that the eEPSC is lower in the control neuron, and vice versa. No more than one paired recording was performed on any given entorhino-hippocampal slice. To elicit inhibitory synaptic responses, the glass monopolar stimulating pipette was placed in the molecular layer of the dentate gyrus. $100 \mathrm{uM} \mathrm{D-APV}$ and $10 \mathrm{uM} \mathrm{NBQX}$ were added to a picrotoxin-free external solution to isolate GABAR-eIPSCs.

For IV rectification analysis, $0.1 \mathrm{mM}$ spermine was added to the internal solution described above for measurement of AMPA receptor-mediated current rectification. Rectification indices were calculated as the normalized glutamateevoked current at $+40 \mathrm{mV}$ over $-70 \mathrm{mV}$, respectively, in presence of $100 \mu \mathrm{M}$ APV to block NMDAR-mediated EPSCs.

For measuring surface AMPAR currents, a picospritzer II (General Valve Co.) was used to puff-apply L-glutamate onto the dendrites of DG granule neurons in the molecular layer or onto the apical dendrites of CA1 pyramidal neurons during recording. Glutamate pulses of $10 \mathrm{~ms}$ were applied to patched neurons held at $-70 \mathrm{mV}$ by a glass pipette. L-glutamate $(25 \mathrm{mM})$ was applied to cells in a solution containing (in $\mathrm{mM}$ ) $\mathrm{NaCl} 140, \mathrm{KCl} 5, \mathrm{MgCl}_{2} 1.4, \mathrm{CaCl}_{2} 1$, EGTA 5 , and $\mathrm{pH}$ adjusted to 7.4. $100 \mathrm{uM} \mathrm{APV}$ was added to isolate AMPAR currents.

For current-clamp recordings, the intracellular solution contained (in $\mathrm{mM}$ ) 130 $\mathrm{KMeSO}_{4}, 10 \mathrm{KCl}, 10$ HEPES, $4 \mathrm{NaCl}, 1$ EGTA, $4 \mathrm{Mg}$-ATP, and $0.3 \mathrm{Na}$-GTP. $500 \mathrm{~ms}$ square current pulses were delivered to neurons held in current-clamp mode. For each recording, current pulse amplitude was increased from 10 to 110 $\mathrm{pA}$ in $10 \mathrm{pA}$ increments. Rheobase values were defined as the minimum injected current required to elicit a single spike.

BSAP97 localization imaging. DG granule neurons in organotypic entorhinohippocampal slice cultures made from P6-P8 rats were biolistically co-transfected with mCherry-tagged $\beta S A P 97$ constructs (wild-type, G344R, or G357S) and the GFP expressing pFUGW- $\beta$ SAP97-miR construct $\sim 18-20 \mathrm{~h}$ after plating. Slices were fixed in $4 \%$ PFA/4\% Sucrose in PBS and washed $3 \mathrm{x}$ with PBS. Slices were further processed with an abbreviated SeeDB-based protocol for imaging $54,84,85$. Images were acquired at DIV7 via multiphoton confocal microscopy (SP8 LIGHTNING Confocal Microscope, Leica). Images were acquired using a $63 \mathrm{x} / 1.4 \mathrm{NA}$ oil immersion objective. Imaris image analysis software (Oxford Instruments) was used to identify and visualize dendritic regions exhibiting the highest $\beta$ SAP97 fluorescent intensity.

Protein modeling. The high-resolution crystal structures of the SAP97 PDZ2 domain (PDB ID: 2AWX, $1.80 \AA$ A resolution; https://www.rcsb.org/structure/
2AWX) and the SAP97 PDZ2 domain in complex with a GluA1 C-terminal peptide (PDB ID: 2G2L, 2.35 $\AA$ resolution) were used to predict the effect of mutations on binding GluAl's PDZ-binding domain. Calculations were performed using ICM molecular modeling software (Molsoft LLC). Our modeling showed that mutation G357S results in the formation of a hydrogen bond between the hydroxyl group of S357 and carbonyl oxygen of I354. Substitution of flexible Glycine to Serine and formation of an additional hydrogen bond reduces the flexibility of the $\beta \mathrm{C}-\alpha \mathrm{A}$ loop. Moreover, this change may affect the conformational changes of $\beta \mathrm{A}-$ $\beta \mathrm{B}$ observed upon peptide binding, as $\beta \mathrm{A}-\beta \mathrm{B}$ and $\beta \mathrm{C}-\alpha \mathrm{A}$ loops are located in close proximity to each other. The G344R mutation was predicted to impact the conformation of $\beta \mathrm{B}-\beta \mathrm{C}$ loop, as $\mathrm{G} 344$ has torsion angles that are not compatible with other amino acid residues.

Behavior-animals and surgery. For all behavior experiments, male Sprague Dawley rats (Envigo, Indianapolis, IN, USA) weighing 300-400 g ( P90) were individually housed in wire-hanging cages in a climate controlled $\left(22-24^{\circ} \mathrm{C}\right)$ environment with a 12:12 h light/dark cycle. Rats were given ad libitum access to water and standard rodent chow (LabDiet 5001, LabDiet, St. Louis, MO). Experiments were performed in accordance with NIH Guidelines for the Care and Use of Laboratory Animals, and all procedures were approved by the Institutional Animal Care and Use Committee of the University of Southern California.

For stereotaxic injection of AAVs for in vivo knockdown of SAP97 expression in the dorsal dentate gyrus (dDG) or in the dorsal CA1, rats were first anesthetized and sedated with a ketamine $(90 \mathrm{mg} / \mathrm{kg}) / \mathrm{xylazine}(2.8 \mathrm{mg} / \mathrm{kg}) /$ acepromazine $(0.72 \mathrm{mg} / \mathrm{kg})$ cocktail. Animals were then shaved and the surgical site was prepped with iodine and ethanol swabs before being placed in a stereotaxic apparatus for stereotaxic injections. $\beta$ SAP97-miR AAV and scrambled-miR AAV were prepared by Vector Biolabs, as described above. AAVs were delivered bilaterally to either the dDG (AP: -3.12 , ML: +/-1.20, DV:-3.9) or the dorsal CA1 (AP: -3.12 , ML: + /$1.50, \mathrm{DV}:-3.0)$ at an injection volume of $200 \mathrm{nl}$ per hemisphere via pressure injections. Injections were administered using a microinfusion pump (Harvard Apparatus, Holliston, MA) connected to a 33-gauge microsyringe injector attached to a PE20 catheter and Hamilton syringe. The flow rate was calibrated and set to $5 \mu \mathrm{l} / \mathrm{min}$. Injectors were left in place for $2 \mathrm{~min}$ to allow for complete infusion of the drug. Behavioral experimental procedures began 21 days post virus injection to allow for transduction and miR expression. Statistical analyses confirmed that the scrambled miR and nonsurgical controls groups did not significantly differ for any behavioral measures, and thus these groups were combined into a single control group for all subsequent analyses. Brains of all animals that underwent behavioral testing were subjected to post hoc immunohistochemical analysis to ensure that data was only included from animals where transduction was restricted to either the dentate gyrus or CA1 region of the hippocampus (see Supplementary Fig. 4)

Novel object in context task. Rats were tested on their episodic contextual memory abilities using the hippocampal-dependent Novel Object in Context (NOIC) task, which was adapted from previous reports ${ }^{70-72,86,87}$. Briefly, each animal received a 5-min session per day in a behavioral box and the box and objects were cleaned with $10 \%$ ETOH between each animal. Rats were habituated on consecutive days to Context 1 , a semi-transparent box $(15$ in $\mathrm{W} \times 24$ in $\mathrm{L} \times 12$ in $\mathrm{H})$ with yellow stripes, or Context 2 , a gray opaque box $(17$ in $\mathrm{W} \times 17$ in $\mathrm{L} \times 16$ in $\mathrm{H}$ ), counterbalanced by group and context order. Following habituation, on day 1 of NOIC, each animal was placed in Context 1 containing a soap dispenser (Object A) and an empty mason jar (Object B) placed on diagonal, equidistant markings with ample space for the rat to circle the objects. Notably, the side the objects were located on (left or right) was counterbalanced by group. The following day (day 2 of NOIC) rats were placed in Context 2 placed in a different room with duplicates of either Object A or Object B. On the test day, day 3 of NOIC, rats were placed again in Context 2, except this time with both Object A and Object B. Depending on the duplicate objects seen on day 2 of NOIC, Object A or Object B on day 3 of NOIC was not a novel object per se, but its placement in Context 2 was novel. Untreated rats will preferentially explore the contextual novel object, an effect that would be disrupted with hippocampal inactivation ${ }^{87}$. On day 1 and day 3 , exploration was hand-scored live by an experimenter blind to group assignments from video recordings using a ceiling Digital USB 2.0 CMOS Camera (Stöelting Co., Wood Dale, IL) and was defined as sniffing or touching the object with the nose or forepaws. Time spent exploring Novel Object in Context/(Time spent exploring Object A + Object B) $] \times 100$ was calculated on both days and the $\%$ shift from baseline was determined by subtracting the value of day 1 from day 3 .

Novel object recognition task. NOR procedures were adapted from Beilharz et al ${ }^{88}$ Briefly, a gray opaque box $(17$ in $\mathrm{W} \times 17$ in $\mathrm{L} \times 16$ in $\mathrm{H})$ was used as an arena and placed in a dimly lit room, achieved by pointing two desk lamps face down on opposite ends of the box. Rats were habituated to the empty arena for $10 \mathrm{~min}$ on the day prior to testing. The novel object and the side on which the novel object was placed were counterbalanced by the group. The test began with a 5-min familiarization phase, where rats were placed in the center of the arena, facing away from the objects, with two identical copies of the same object to explore. The objects were either two identical $12 \mathrm{oz}$. cans or two identical stemless wine glasses, counterbalanced by treatment group. The objects were chosen based on 
preliminary studies which determined that they are equally preferred by Sprague Dawley rats. Animals were then removed from the arena and placed in the home cage for $5 \mathrm{~min}$. Meanwhile, the arena and objects were cleaned with $10 \%$ ethanol solution, and one of the objects in the arena was replaced with a different one (either the $12 \mathrm{oz}$. can or stemless wine glass, whichever the animal had not previously seen, i.e., the "novel object"). Then, the animals were again placed in the center of the arena and allowed to explore for $3 \mathrm{~min}$. Time spent exploring the objects were hand-scored live from videos recorded from a ceiling Digital USB 2.0 CMOS Camera (Stöelting Co., Wood Dale, IL). Then, the Recognition index [novel object exploration (s)/[novel object exploration $(\mathrm{s})+$ familiar object exploration (s)] was calculated for each animal, with higher values on this index indicating greater exploration of the novel object.

Zero maze task. Rodents were tested for potential anxiety-like effects associated with injecting the $\beta S A P 97-\mathrm{miR}$ AAV into either the dorsal dentate gyrus or dorsal CA1 using a Zero Maze. The Zero Maze is an elevated circular platform $(63.5 \mathrm{~cm}$ height, $116.8 \mathrm{~cm}$ external diameter) with two closed zones and two open zones, all of which are equal in length. The closed zones are enclosed with $17.5 \mathrm{~cm}$ high walls whereas the open zones have only $3 \mathrm{~cm}$ high curbs. Any-Maze software (Stöelting Co., Wood Dale, IL) was used to video record the animals and analyze time spent in the open zones of the maze. Animals were placed in the maze for a single 5 min session and after each session, the apparatus was cleaned with $10 \%$ ethanol.

Open field. Open field measures both general locomotor activity and anxiety-like behavior in the rat. Here, a large gray bin, $60 \mathrm{~cm}(\mathrm{~L}) \times 56 \mathrm{CM}(\mathrm{W})$ was used as an arena and was placed under diffuse even lighting $(30 \mathrm{~lx})$. A center zone $(19 \mathrm{~cm} \mathrm{~L} \times$ $17.5 \mathrm{~cm} \mathrm{~W}$ ) was identified and marked using Any-Maze software (Stoelting Co., Wood Dale, IL) and a USB camera directly overhead connected to Any-Maze software tracked the movement of the animals. Animals were placed in the center of the box and allowed to explore the arena for $10 \mathrm{~min}$, with the dependent variable being the total distance traveled $(\mathrm{m})$. The apparatus was cleaned with $10 \%$ ethanol after each rat was tested.

Statistical analysis. Paired electrophysiological recordings of eEPSC amplitudes were analyzed using paired two-tailed $T$ tests. Two-sample two-tailed $T$ tests were used to compare electrophysiological data across independent conditions. P-values of $<0.05$ were considered statistically significant (KaleidaGraph). Coefficient of Variation (CV) analysis was performed on AMPAR-eEPSCs by comparing the change in eEPSC variance with the change in mean amplitude as previously described $^{54,55,57,60,76,77,89,90}$. CV was calculated as $\mathrm{SD} / M(\mathrm{SD}=$ standard deviation; $M=$ mean). The SD and $M$ were measured, normalized and plotted for a concurrent set of stimuli from a control and its neighboring transfected cell. It has been shown both theoretically and experimentally that changes in $\mathrm{CV}^{-2}\left(M^{2} / \mathrm{SD}^{2}\right)$ are independent of quantal size but vary in a predictable manner with quantal content: number of release sites $n \times$ presynaptic release probability, $\mathrm{Pr} ; \mathrm{CV}^{-2}=n \operatorname{Pr} /(1-$ $\mathrm{Pr})^{89-92}$. CV analysis is presented as scatter plots with $\mathrm{CV}^{-2}$ values calculated for transfected cell/control cell pairs on the $y$-axis and mean eEPSC amplitude values of transfected/cell control cell pairs on the $x$-axis. Filled circles represent the mean \pm SEM of the entire dataset. Filled circles that fall on or near the $45^{\circ}(y=x)$ line reflect changes in quantal content while values approaching the horizontal $(y=1)$ reflect a change in quantal size. Slice immunolabeling results were replicated using slices from at least five different animals. Quantified biochemical results were replicated at least three times using independent samples. For behavioral experiments, NOIC and NOR were analyzed using a multi-factor ANOVA (Statistica Version 7; Statsoft) with the surgical group, training squad, and novel object assignment as between-subjects variables. All other behavioral tasks were analyzed using a Student's two-tailed two-sample $T$ test performed using Statistica V7. The Grubbs test for outliers was used as pre-established exclusion criteria (Prism 8). $P$ values of $<0.05$ were considered statistically significant. Error bars represent standard error of the mean measurement. Sample sizes in the present study are similar to those reported in the literature ${ }^{59,93}$ for electrophysiological recordings of eEPSC amplitudes and, for behavioral tasks, sample size was chosen based on a priori power analyses (conducted in Statistica V7) to ensure sufficient power to detect a pre-specified effect size. For biochemical data, two-sample $T$ tests were used to compare experimental conditions. Additional statistical information for all experiments performed is provided in the Source Data file.

Reporting summary. Further information on research design is available in the Nature Research Reporting Summary linked to this article.

\section{Data availability}

All data supporting the findings of this study are provided within the paper and its supplementary information. A source data file is provided with this paper. All additional information will be made available upon reasonable request to the authors.

Received: 21 January 2021; Accepted: 5 January 2022;

Published online: 10 February 2022

\section{References}

1. Yun, S., Reynolds, R. P., Masiulis, I. \& Eisch, A. J. Re-evaluating the link between neuropsychiatric disorders and dysregulated adult neurogenesis. Nat. Med. 22, 1239-1247 (2016).

2. Kahn, R. S. et al. Schizophrenia. Nat. Rev. Dis. Prim. 1, 15067 (2015).

3. Toyooka, K. et al. Selective reduction of a PDZ protein, SAP-97, in the prefrontal cortex of patients with chronic schizophrenia. J. Neurochem. 83, 797-806 (2002).

4. Kushima, I. et al. Comparative analyses of copy-number variation in autism spectrum disorder and schizophrenia reveal etiological overlap and biological insights. Cell Rep. 24, 2838-2856 (2018).

5. Soler, J. et al. Genetic variability in scaffolding proteins and risk for schizophrenia and autism-spectrum disorders: a systematic review. $J$. Psychiatry Neurosci. 43, 223-244 (2018).

6. Carroll, L. S. et al. Mutation screening of the 3 q29 microdeletion syndrome candidate genes DLG1 and PAK2 in schizophrenia. Am. J. Med. Genet. B: Neuropsychiatr. Genet. 156B, 844-849 (2011).

7. Purcell, S. M. et al. A polygenic burden of rare disruptive mutations in schizophrenia. Nature 506, 185-190 (2014).

8. Mulle, J. G. et al. Microdeletions of $3 \mathrm{q} 29$ confer high risk for schizophrenia Am. J. Hum. Genet. 87, 229-236 (2010).

9. Mulle, J. G. The $3 \mathrm{q} 29$ deletion confers $>40$-fold increase in risk for schizophrenia. Mol. Psychiatry 20, 1028-1029 (2015).

10. Sato, J., Shimazu, D., Yamamoto, N. \& Nishikawa, T. An association analysis of synapse-associated protein 97 (SAP97) gene in schizophrenia. J. Neural Transm. (Vienna) 115, 1355-1365 (2008).

11. Uezato, A. et al. Further evidence for a male-selective genetic association of synapse-associated protein 97 (SAP97) gene with schizophrenia. Behav. Brain Funct. 8, 2 (2012).

12. Xing, J. et al. Resequencing and association analysis of Six PSD-95-related genes as possible susceptibility genes for schizophrenia and autism spectrum disorders. Sci. Rep. 6, 27491 (2016).

13. Marshall, C. R. et al. Contribution of copy number variants to schizophrenia from a genome-wide study of 41,321 subjects. Nat. Genet. 49, 27-35 (2017).

14. Fromer, M. et al. De novo mutations in schizophrenia implicate synaptic networks. Nature 506, 179-184 (2014).

15. Won, S., Levy, J. M., Nicoll, R. A. \& Roche, K. W. MAGUKs: multifaceted synaptic organizers. Curr. Opin. Neurobiol. 43, 94-101 (2017).

16. Leonard, A. S., Davare, M. A., Horne, M. C., Garner, C. C. \& Hell, J. W. SAP97 is associated with the alpha-amino-3-hydroxy-5-methylisoxazole-4-propionic acid receptor GluR1 subunit. J. Biol. Chem. 273, 19518-19524 (1998).

17. Sans, N. et al. Synapse-associated protein 97 selectively associates with a subset of AMPA receptors early in their biosynthetic pathway. J. Neurosci. 21, 7506-7516 (2001).

18. Kim, C. H. et al. Persistent hippocampal CA1 LTP in mice lacking the C-terminal PDZ ligand of GluR1. Nat. Neurosci. 8, 985-987 (2005).

19. Zhou, W. et al. GluR1 controls dendrite growth through its binding partner, SAP97. J. Neurosci. 28, 10220-10233 (2008).

20. Cai, C., Coleman, S. K., Niemi, K. \& Keinanen, K. Selective binding of synapse-associated protein 97 to GluR-A alpha-amino-5-hydroxy-3-methyl-4isoxazole propionate receptor subunit is determined by a novel sequence motif. J. Biol. Chem. 277, 31484-31490 (2002).

21. Granger, A. J., Shi, Y., Lu, W., Cerpas, M. \& Nicoll, R. A. LTP requires a reserve pool of glutamate receptors independent of subunit type. Nature 493 495-500 (2013).

22. Nakagawa, T. et al. Quaternary structure, protein dynamics, and synaptic function of SAP97 controlled by L27 domain interactions. Neuron 44, 453-467 (2004).

23. Schnell, E. et al. Direct interactions between PSD-95 and stargazin control synaptic AMPA receptor number. Proc. Natl Acad. Sci. USA 99, 13902-13907 (2002).

24. Schluter, O. M., Xu, W. \& Malenka, R. C. Alternative N-terminal domains of PSD-95 and SAP97 govern activity-dependent regulation of synaptic AMPA receptor function. Neuron 51, 99-111 (2006).

25. Li, D. et al. SAP97 directs NMDA receptor spine targeting and synaptic plasticity. J. Physiol. 589, 4491-4510 (2011).

26. Ehrlich, I., Klein, M., Rumpel, S. \& Malinow, R. PSD-95 is required for activity-driven synapse stabilization. Proc. Natl Acad. Sci. USA 104, 4176-4181 (2007).

27. Rumbaugh, G., Sia, G. M., Garner, C. C. \& Huganir, R. L. Synapse-associated protein-97 isoform-specific regulation of surface AMPA receptors and synaptic function in cultured neurons. J. Neurosci. 23, 4567-4576 (2003).

28. Howard, M. A., Elias, G. M., Elias, L. A., Swat, W. \& Nicoll, R. A. The role of SAP97 in synaptic glutamate receptor dynamics. Proc. Natl Acad. Sci. USA 107, 3805-3810 (2010).

29. Volk, L., Chiu, S. L., Sharma, K. \& Huganir, R. L. Glutamate synapses in human cognitive disorders. Annu. Rev. Neurosci. 38, 127-149 (2015). 
30. Das, T., Ivleva, E. I., Wagner, A. D., Stark, C. E. \& Tamminga, C. A. Loss of pattern separation performance in schizophrenia suggests dentate gyrus dysfunction. Schizophr. Res 159, 193-197 (2014).

31. Tamminga, C. A., Stan, A. D. \& Wagner, A. D. The hippocampal formation in schizophrenia. Am. J. Psychiatry 167, 1178-1193 (2010).

32. Kawano, M. et al. Hippocampal subfield volumes in first episode and chronic schizophrenia. PLoS ONE 10, e0117785 (2015).

33. Ota, M. et al. Structural differences in hippocampal subfields among schizophrenia patients, major depressive disorder patients, and healthy subjects. Psychiatry Res. Neuroimaging 259, 54-59 (2017).

34. Falkai, P. et al. Decreased oligodendrocyte and neuron number in anterior hippocampal areas and the entire hippocampus in schizophrenia: a stereological postmortem study. Schizophr. Bull. 42, S4-S12 (2016).

35. Kirov, I. I. et al. In vivo 7 Tesla imaging of the dentate granule cell layer in schizophrenia. Schizophr. Res. 147, 362-367 (2013).

36. Jaffe, A. E. et al. Profiling gene expression in the human dentate gyrus granule cell layer reveals insights into schizophrenia and its genetic risk. Nat. Neurosci. 23, 510-519 (2020).

37. Nakahara, S. et al. Dentate gyrus volume deficit in schizophrenia. Psychol. Med. 50, 1267-1277 (2019)

38. Tavitian, A., Song, W. \& Schipper, H. M. Dentate gyrus immaturity in schizophrenia. Neuroscientist 25, 528-547 (2019).

39. Berron, D. et al. Strong evidence for pattern separation in human dentate gyrus. J. Neurosci. 36, 7569-7579 (2016).

40. Servan-Schreiber, D., Cohen, J. D. \& Steingard, S. Schizophrenic deficits in the processing of context. A test of a theoretical model. Arch. Gen. Psychiatry 53, 1105-1112 (1996).

41. Hemsley, D. R. The schizophrenic experience: taken out of context? Schizophr. Bull. 31, 43-53 (2005).

42. Bazin, N., Perruchet, P., Hardy-Bayle, M. C. \& Feline, A. Context-dependent information processing in patients with schizophrenia. Schizophr. Res. 45 93-101 (2000)

43. Rizzo, L., Danion, J. M., van der Linden, M. \& Grange, D. Patients with schizophrenia remember that an event has occurred, but not when. Br. J. Psychiatry 168, 427-431 (1996).

44. Rizzo, L., Danion, J. M., Van Der Linden, M., Grange, D. \& Rohmer, J. G. Impairment of memory for spatial context in schizophrenia. Neuropsychology 10, 376-384 (1996).

45. Waters, F. A., Maybery, M. T., Badcock, J. C. \& Michie, P. T. Context memory and binding in schizophrenia. Schizophr. Res. 68, 119-125 (2004).

46. Doughty, O. J., Done, D. J., Lawrence, V. A., Al-Mousawi, A. \& Ashaye, K. Semantic memory impairment in schizophrenia-deficit in storage or access of knowledge? Schizophr. Res. 105, 40-48 (2008).

47. Murty, V. P. et al. Differential patterns of contextual organization of memory in first-episode psychosis. NPJ Schizophr. 4, 3 (2018).

48. Aleman, A., Hijman, R., de Haan, E. H. \& Kahn, R. S. Memory impairment in schizophrenia: a meta-analysis. Am. J. Psychiatry 156, 1358-1366 (1999).

49. Greenland-White, S. E., Ragland, J. D., Niendam, T. A., Ferrer, E. \& Carter, C. S. Episodic memory functions in first episode psychosis and clinical high risk individuals. Schizophr. Res. 188, 151-157 (2017).

50. Lewandowski, K. E., Cohen, B. M. \& Ongur, D. Evolution of neuropsychological dysfunction during the course of schizophrenia and bipolar disorder. Psychol. Med. 41, 225-241 (2011).

51. Doughty, O. J. \& Done, D. J. Is semantic memory impaired in schizophrenia? A systematic review and meta-analysis of 91 studies. Cogn. Neuropsychiatry 14, 473-509 (2009).

52. Libby, L. A., Yonelinas, A. P., Ranganath, C. \& Ragland, J. D. Recollection and familiarity in schizophrenia: a quantitative review. Biol. Psychiatry 73, 944-950 (2013).

53. Elias, G. M., Elias, L. A., Apostolides, P. F., Kriegstein, A. R. \& Nicoll, R. A. Differential trafficking of AMPA and NMDA receptors by SAP102 and PSD95 underlies synapse development. Proc. Natl Acad. Sci. USA 105, 20953-20958 (2008)

54. Sadybekov, A., Tian, C., Arnesano, C., Katritch, V. \& Herring, B. E. An autism spectrum disorder-related de novo mutation hotspot discovered in the GEF1 domain of Trio. Nat. Commun. 8, 601 (2017).

55. Tian, C. et al. An intellectual disability-related missense mutation in Rac1 prevents LTP induction. Front Mol. Neurosci. 11, 223 (2018).

56. Paskus, J. D. et al. Synaptic kalirin-7 and trio interactomes reveal a GEF protein-dependent neuroligin-1 mechanism of action. Cell Rep. 29, 2944-2952 e2945 (2019)

57. Rao, S., Kay, Y. \& Herring, B. E. Tiam1 is critical for glutamatergic synapse structure and function in the hippocampus. J. Neurosci. 39, 9306-9315 (2019).

58. Herring, B. E. et al. Cornichon proteins determine the subunit composition of synaptic AMPA receptors. Neuron 77, 1083-1096 (2013)

59. Herring, B. E. \& Nicoll, R. A. Kalirin and trio proteins serve critical roles in excitatory synaptic transmission and LTP. Proc. Natl Acad. Sci. USA 113 2264-2269 (2016).
60. Levy, J. M., Chen, X., Reese, T. S. \& Nicoll, R. A. Synaptic consolidation normalizes AMPAR quantal size following MAGUK loss. Neuron 87, 534-548 (2015).

61. Waites, C. L. et al. Synaptic SAP97 isoforms regulate AMPA receptor dynamics and access to presynaptic glutamate. J. Neurosci. 29, 4332-4345 (2009).

62. Park, P. et al. The role of calcium-permeable AMPARs in long-term potentiation at principal neurons in the rodent hippocampus. Front Synaptic Neurosci. 10, 42 (2018)

63. Elias, G. M. et al. Synapse-specific and developmentally regulated targeting of AMPA receptors by a family of MAGUK scaffolding proteins. Neuron 52, 307-320 (2006).

64. El-Husseini, A. E., Schnell, E., Chetkovich, D. M., Nicoll, R. A. \& Bredt, D. S. PSD-95 involvement in maturation of excitatory synapses. Science $\mathbf{2 9 0}$, 1364-1368 (2000)

65. Beique, J. C. \& Andrade, R. PSD-95 regulates synaptic transmission and plasticity in rat cerebral cortex. J. Physiol. 546, 859-867 (2003).

66. Ling, W. et al. Immunolocalization of NR1, NR2A, and PSD-95 in rat hippocampal subregions during postnatal development. Acta Histochem. 114, 285-295 (2012).

67. Cuthbert, P. C. et al. Synapse-associated protein 102/dlgh3 couples the NMDA receptor to specific plasticity pathways and learning strategies. J. Neurosci. 27, 2673-2682 (2007).

68. Su, D. et al. Dynamic SAP102 expression in the hippocampal subregions of rats and APP/PS1 mice of various ages. J. Anat. 232, 987-996 (2018).

69. Talamini, L. M., de Haan, L., Nieman, D. H., Linszen, D. H. \& Meeter, M. Reduced context effects on retrieval in first-episode schizophrenia. PLoS One 5, e10356 (2010).

70. Davis, E. A. et al. Ghrelin signaling affects feeding behavior, metabolism, and memory through the vagus nerve. Curr. Biol. 30, 4510-4518.e6 (2020).

71. Suarez, A. N. et al. Gut vagal sensory signaling regulates hippocampus function through multi-order pathways. Nat. Commun. 9, 2181 (2018)

72. Noble, E. E. et al. Early-life sugar consumption affects the rat microbiome independently of obesity. J. Nutr. 147, 20-28 (2017)

73. Jackson, A. C. \& Nicoll, R. A. The expanding social network of ionotropic glutamate receptors: TARPs and other transmembrane auxiliary subunits. Neuron 70, 178-199 (2011).

74. von Ossowski, I. et al. Crystal structure of the second PDZ domain of SAP97 in complex with a GluR-A C-terminal peptide. FEBS J. 273, 5219-5229 (2006)

75. Lussier, M. P., Gu, X., Lu, W. \& Roche, K. W. Casein kinase 2 phosphorylates GluA1 and regulates its surface expression. Eur. J. Neurosci. 39, 1148-1158 (2014).

76. Gray, J. A. et al. Distinct modes of AMPA receptor suppression at developing synapses by GluN2A and GluN2B: single-cell NMDA receptor subunit deletion in vivo. Neuron 71, 1085-1101 (2011).

77. Tian, C., Paskus, J. D., Fingleton, E., Roche, K. W. \& Herring, B. E. Autism spectrum disorder/intellectual disability-associated mutations in trio disrupt neuroligin 1-mediated synaptogenesis. J. Neurosci. 41, 7768-7778 (2021)

78. Wyszynski, M. et al. Competitive binding of alpha-actinin and calmodulin to the NMDA receptor. Nature 385, 439-442 (1997).

79. Funke, L., Dakoji, S. \& Bredt, D. S. Membrane-associated guanylate kinases regulate adhesion and plasticity at cell junctions. Annu. Rev. Biochem. 74, 219-245 (2005).

80. Lambert, J. T., Hill, T. C., Park, D. K., Culp, J. H. \& Zito, K. Protracted and asynchronous accumulation of PSD95-family MAGUKs during maturation of nascent dendritic spines. Dev. Neurobiol. 77, 1161-1174 (2017).

81. Bonnici, B. \& Kapfhammer, J. P. Modulators of signal transduction pathways can promote axonal regeneration in entorhino-hippocampal slice cultures. Eur. J. Pharm. 612, 35-40 (2009)

82. Prang, P., Del Turco, D. \& Kapfhammer, J. P. Regeneration of entorhinal fibers in mouse slice cultures is age dependent and can be stimulated by NT-4, GDNF, and modulators of G-proteins and protein kinase C. Exp. Neurol. 169, 135-147 (2001).

83. Stoppini, L., Buchs, P. A. \& Muller, D. A simple method for organotypic cultures of nervous tissue. J. Neurosci. Methods 37, 173-182 (1991).

84. Ke, M. T., Fujimoto, S. \& Imai, T. SeeDB: a simple and morphologypreserving optical clearing agent for neuronal circuit reconstruction. Nat. Neurosci. 16, 1154-1161 (2013).

85. Kay, Y. \& Herring, B. E. An optogenetic method for investigating presynaptic molecular regulation. Sci. Rep. 11, 11329 (2021).

86. Balderas, I. et al. The consolidation of object and context recognition memory involve different regions of the temporal lobe. Learn Mem. 15, 618-624 (2008).

87. Martinez, M. C., Villar, M. E., Ballarini, F. \& Viola, H. Retroactive interference of object-in-context long-term memory: role of dorsal hippocampus and medial prefrontal cortex. Hippocampus 24, 1482-1492 (2014).

88. Beilharz, J. E., Maniam, J. \& Morris, M. J. Short exposure to a diet rich in both fat and sugar or sugar alone impairs place, but not object recognition memory in rats. Brain Behav. Immun. 37, 134-141 (2014). 
89. Bekkers, J. M. \& Stevens, C. F. Presynaptic mechanism for long-term potentiation in the hippocampus. Nature 346, 724-729 (1990).

90. Malinow, R. \& Tsien, R. W. Presynaptic enhancement shown by whole-cell recordings of long-term potentiation in hippocampal slices. Nature 346, 177-180 (1990).

91. Del Castillo, J. \& Katz, B. Quantal components of the end-plate potential. J. Physiol. 124, 560-573 (1954).

92. Xiang, Z., Greenwood, A. C., Kairiss, E. W. \& Brown, T. H. Quantal mechanism of long-term potentiation in hippocampal mossy-fiber synapses. $J$. Neurophysiol. 71, 2552-2556 (1994).

93. Incontro, S. et al. The CaMKII/NMDA receptor complex controls hippocampal synaptic transmission by kinase-dependent and independent mechanisms. Nat. Commun. 9, 2069 (2018).

\section{Acknowledgements}

The authors thank members of the Herring lab, Kanoski lab, Katritch lab and Dr. Don Arnold for helpful feedback and comments during the study. We also thank Brendan Miller and Manuel Cerpas for expert technical assistance. Research was supported by the National Institute of Mental Health (MH103398 to B.E.H.), the National Institute of Neurological Disorders and Stroke (NS112480 to B.E.H.) and the Simons and McKnight Foundations (to B.E.H.).

\section{Author contributions}

Y.K. and B.E.H. designed the project. Y.K., L.T., E.A.D, C.T., L.D-S., A.N.P., S.E.K., and B.E.H. collected and analyzed the data. A.S. performed the structural modeling. Y.K., V.K., S.E.K., and B.E.H. wrote the paper with input from all authors.

\section{Competing interests}

The authors declare no competing interests.

\section{Additional information}

Supplementary information The online version contains supplementary material available at https://doi.org/10.1038/s41467-022-28430-5.

Correspondence and requests for materials should be addressed to Bruce E. Herring.

Peer review information Nature Communications thanks Andrew Penn, Daniel Choquet and the other anonymous reviewer(s) for their contribution to the peer review this work.

Reprints and permission information is available at http://www.nature.com/reprints

Publisher's note Springer Nature remains neutral with regard to jurisdictional claims in published maps and institutional affiliations.

Open Access This article is licensed under a Creative Commons Attribution 4.0 International License, which permits use, sharing, adaptation, distribution and reproduction in any medium or format, as long as you give appropriate credit to the original author(s) and the source, provide a link to the Creative Commons license, and indicate if changes were made. The images or other third party material in this article are included in the article's Creative Commons license, unless indicated otherwise in a credit line to the material. If material is not included in the article's Creative Commons license and your intended use is not permitted by statutory regulation or exceeds the permitted use, you will need to obtain permission directly from the copyright holder. To view a copy of this license, visit http://creativecommons.org/ licenses/by/4.0/.

(C) The Author(s) 2022 